\title{
Derived category of moduli of pointed curves. I
}

\author{
Ana-Maria Castravet and Jenia Tevelev
}

\begin{abstract}
This is the first paper in a sequence devoted to the derived category of moduli spaces of curves of genus 0 with marked points. We develop several approaches to describe it equivariantly with respect to the action of the symmetric group permuting marked points. We construct an equivariant full exceptional collection on the Losev-Manin space which categorifies derangements.
\end{abstract}

\section{Introduction}

The special feature of moduli spaces of curves with marked points is the action of the symmetric group permuting marked points, and our goal is to exhibit this action in the description of the derived category. One can think about the derived category as an enhanced cohomological invariant. Although there are many papers in the literature computing cohomology of $\bar{M}_{0, n}$, the moduli space of curves with $n$ marked points, as a module over the symmetric group (for example, [Get95, BM13]), the equivariant Euler-Poincaré polynomial is expressed as an alternating sum, which therefore has no obvious categorification. On the other hand, it is often easy to get some description of the derived category which, however, does not respect the group action. For example, it is obvious that $D^{b}\left(\bar{M}_{0, n}\right)$ has a full exceptional collection. Indeed, $\bar{M}_{0, n}$ has a Kapranov model as an iterated blow-up of $\mathbb{P}^{n-3}$ in $n-1$ points followed by the blow-up of $\left(\begin{array}{c}n-1 \\ 2\end{array}\right)$ proper transforms of lines connecting points, etc. With a little work, Orlov's theorem on the derived category of the blow-up (see Section 3) gives a full exceptional collection. However, Kapranov's model is not unique: it depends on the choice of the $\psi$ class, that is, the choice of a marking, and therefore this collection is not preserved by $S_{n}$ (it is preserved only by $S_{n-1}$ ). The derived categories of $\bar{M}_{0, n}$ and related Hassett spaces and GIT quotients have been studied in [BFK19] and [MS13], although not from the equivariant perspective.

Question 1.1. Is there a full exceptional $S_{n}$-invariant collection on $\bar{M}_{0, n}$ ?

This question of D. Orlov, communicated to us by A. Kuznetsov, will be investigated in detail in the second paper in the series. Note that a striking and unexpected corollary of its existence is that the K-group $K_{0}\left(\bar{M}_{0, n}\right)$ is a permutation representation of $S_{n}$. As a motivation, one can argue that since $\bar{M}_{0, n}$ is smooth over $\operatorname{Spec} \mathbb{Z}$, maybe it is somehow "defined over $\mathbb{F}_{1}$ ",

Received 11 October 2017, accepted in final form 4 March 2020.

2010 Mathematics Subject Classification 14C15, 14D22, 14H10, 14M99, 16E20, 18F30, 20C30, 05A19.

Keywords: exceptional collections, stable rational curves, moduli spaces of weighted stable curves, Losev-Manin spaces.

This journal is (C) Foundation Compositio Mathematica 2020. This article is distributed with Open Access under the terms of the Creative Commons Attribution Non-Commercial License, which permits non-commercial reuse, distribution, and reproduction in any medium, provided that the original work is properly cited. For commercial re-use, please contact the Foundation Compositio Mathematica.

The first-named author was supported by NSF grants DMS-1529735 and DMS-1701752. The second-named author was supported by NSF grants DMS-1303415 and DMS-1701704. 


\section{DERIVED CATEGORY OF MODUli OF POINTED CURVES. I}

and therefore the same should be true of its K-theory as an $S_{n}$-module, and so perhaps it should be a permutation representation.

In this paper, we suggest two general strategies which may have other applications and provide an answer for the Losev-Manin space [LM00].

One approach, which justifies why we consider the case of Losev-Manin spaces, is based on an equivariant version of Orlov's theorem on blow-ups (Section 2) and inspired by the work of Bergström and Minabe in [BM13].

Let $X$ be a smooth projective variety, and let $Y_{1}, \ldots, Y_{n} \subseteq X$ be smooth transversal subvarieties of codimension $l$. For any subset $I \subseteq\{1, \ldots, n\}$, we denote the intersection $\cap_{i \in I} Y_{i}$ by $Y_{I}$. In particular, $Y_{\emptyset}=X$. Let $q: \tilde{X} \rightarrow X$ be an iterated blow-up of (proper transforms of) $Y_{1}, \ldots, Y_{n}$. In addition, let $G$ be a finite group acting on $X$ permuting $Y_{1}, \ldots, Y_{n}$. Then $G$ also acts on $\tilde{X}$, and the morphism $q$ is $G$-equivariant. Let $G_{I} \subseteq G$ be a normalizer of $Y_{I}$ for each subset $I \subseteq\{1, \ldots, n\}$ (in particular, $G_{\emptyset}=G$ ). We show in Lemma 2.3 that if $D^{b}\left(Y_{I}\right)$ admits a full

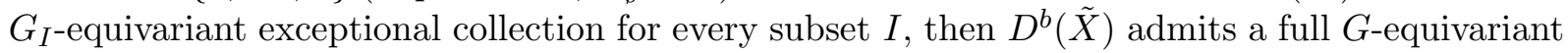
exceptional collection.

Next we generalize an inductive computation given in [BM13] of the equivariant EulerPoincaré polynomial of $\bar{M}_{0, n}$. In the derived category setting, it gives the following theorem. Fix integers $l \geqslant 1$ and $0 \leqslant k \leqslant n$. For a weight

$$
\mathbf{a}=\left(1, \ldots, 1, \frac{1}{l}, \ldots, \frac{1}{l}\right)
$$

(with $k$ copies of 1 and $n-k$ copies of $1 / l$ ), let $\bar{M}_{k, l}^{n}$ be the Hassett moduli space [Has03] of a-weighted stable rational curves. For example, $\bar{M}_{0,1}^{n} \simeq \bar{M}_{0, n}$, and $\bar{M}_{0,\lfloor(n-1) / 2\rfloor}^{n}$ is a symmetric GIT quotient $\left(\mathbb{P}^{1}\right)^{n} / / \mathrm{PGL}_{2}$ if $n$ is odd and its Kirwan resolution if $n$ is even.

THEOREM 1.2. If $\bar{M}_{k, r(n, k)}^{n}$ admits a full $\left(S_{k} \times S_{n-k}\right)$-equivariant exceptional collection for every $n$ and every $0 \leqslant k \leqslant n-3$, then $\bar{M}_{0, n}$ admits a full $S_{n}$-equivariant exceptional collection for every $n$. Here

$$
r(n, k):= \begin{cases}\lfloor(n-1) / 2\rfloor & \text { if } k=0, \\ n-2 & \text { if } k=1, \\ n-k & \text { if } k \geqslant 2 .\end{cases}
$$

Concretely, we need the following spaces:

- the symmetric GIT quotient and its Kirwan resolution, which will be studied in the sequel to this paper

- $\bar{M}_{1, n-2}^{n}$, which is isomorphic to $\mathbb{P}^{n-3}$ via the Kapranov map (we can take any standard exceptional collection on $\mathbb{P}^{n-3}$, for example $\left.\mathcal{O}, \ldots, \mathcal{O}(n-3)\right)$

- $\bar{M}_{2, n-2}^{n}$ (this is the Losev-Manin space studied in this paper)

- spaces $\bar{M}_{k, n-k}^{n}$ for $k>2$ (these spaces are still too complicated for the calculations of the derived category, and in the sequel to this paper, we will investigate their further equivariant reductions).

We now discuss another strategy, which is the one we will use in this paper for the case of the Losev-Manin spaces $\overline{L M}_{n}$. We start with an example.

Example 1.3. Unlike $\bar{M}_{0,5}$, which has five Kapranov models and therefore five Orlov-style exceptional collections, the 2-dimensional Losev-Manin space, which we denote by $\overline{L M}_{3}$ in this paper 


\section{A.-M. Castravet and J. Tevelev}

(see below), has only two non-trivial $\psi$-classes $\psi_{0}$ and $\psi_{\infty}$, realizing it as $\mathbb{P}^{2}$ blown-up at three points $p_{1}, p_{2}, p_{3}$ in two ways, related by the Cremona involution. The corresponding exceptional collection invariant under all automorphisms has three blocks and consists of line bundles

$$
\left\{-\psi_{0},-\psi_{\infty}\right\}, \quad\left\{\pi_{1}^{*} \mathcal{O}(-1), \pi_{2}^{*} \mathcal{O}(-1), \pi_{3}^{*} \mathcal{O}(-1)\right\}, \quad \mathcal{O},
$$

where $\pi_{i}: \overline{L M}_{3} \rightarrow \overline{L M}_{2} \simeq \mathbb{P}^{1}$ is a forgetful map, which can be thought of as a linear projection $\mathbb{P}^{2} \rightarrow \mathbb{P}^{1}$ from the point $p_{i}$.

The last four line bundles in (1.1) are pull-backs under forgetful maps, but the first two have a trivial derived pushforward by any forgetful map. To study situations of this sort more systematically, we introduce an inclusion-exclusion principle in triangulated categories (see Lemma 3.6) and its application in the following set-up.

Definition 1.4. Given a collection of morphisms of smooth projective varieties $\pi_{i}: X \rightarrow X_{i}$ for $i \in I$, we call an object $E \in D^{b}(X)$ cuspidal $^{1}$ if

$$
R \pi_{i *} E=0 \quad \text { for every } i \in I \text {. }
$$

The cuspidal block is the full triangulated subcategory of cuspidal objects

$$
D_{\text {cusp }}^{b}(X) \subset D^{b}(X) .
$$

Philosophically, the cuspidal block captures information about the derived category not already encoded in $D^{b}\left(X_{i}\right)$ for $i \in I$. We show in Theorem 3.5 that under quite general assumptions, $D_{\text {cusp }}^{b}(X)$ is an admissible subcategory and in fact the first block in the "inclusion-exclusion" semi-orthogonal decomposition of $D^{b}(X)$. In our applications, morphisms $\pi_{i}$ are forgetful maps such as $\bar{M}_{0, n} \rightarrow \bar{M}_{0, n-1}$, and thus an $S_{n}$-equivariant description of $D^{b}(X)$ can be reduced to an $S_{n}$-equivariant description of $D_{\text {cusp }}^{b}(X)$.

Question 1.5. Find a full $S_{n}$-invariant exceptional collection in the cuspidal block $D_{\text {cusp }}^{b}\left(\bar{M}_{0, n}\right)$ with respect to all the forgetful maps $\bar{M}_{0, n} \rightarrow \bar{M}_{0, n-1}$.

An answer to Question 1.5 together with Proposition 1.6 (an application of Theorem 3.5) will therefore answer Question 1.1.

Proposition 1.6. We write $\bar{M}_{N} \simeq \bar{M}_{0, n}$ for the moduli space of stable rational curves with points marked by any $n$-element set $N$. Then $D^{b}\left(\bar{M}_{N}\right)$ admits a semi-orthogonal decomposition

$$
D^{b}\left(\bar{M}_{N}\right)=\left\langle D_{\text {cusp }}^{b}\left(\bar{M}_{N}\right),\left\{\pi_{K}^{*} D_{\text {cusp }}^{b}\left(\bar{M}_{N \backslash K}\right)\right\}_{K \subset N}, \mathcal{O}\right\rangle,
$$

where $K$ runs over subsets with $1 \leqslant|K| \leqslant n-4$ in the order of increasing cardinality $|K|$ and $\pi_{K}: \bar{M}_{N} \rightarrow \bar{M}_{N \backslash K}$ is the map that forgets markings in $K$.

We mention the answer to Question 1.5 in the first few small $n$ cases.

Example 1.7. Let $T(-\log )$ be the rank $n-3$ vector bundle on $\bar{M}_{0, n}$ of vector fields tangent to its (normal crossing) boundary divisor. It is easy to deduce from the results of [KT09] that $T(-\log )$ is an exceptional vector bundle and an element of $D_{\text {cusp }}^{b}\left(\bar{M}_{0, n}\right)$ for every $n$. This fact, which we view as a manifestation of rigidity of $\bar{M}_{0, n}$, was one of our original motivations for writing this paper. For small $n$, the category $D_{\text {cusp }}^{b}\left(\bar{M}_{0, n}\right)$ has the following full $S_{n}$-equivariant exceptional collection:

${ }^{1}$ The terminology (suggested to us by A. Oblomkov) comes from cuspidal representations of representation theory. When considering a single morphism, the cuspidal block is sometimes known as the null-category. 


\section{DERIVED CATEGORY OF MODUli OF POINTED CURVES. I}

- $n=4: T(-\log )$ (one object)

- $n=5: T(-\log )$ (one object)

- $n=6: \mathcal{O}_{\mathbb{P}^{1} \times \mathbb{P}^{1}}(-1,-1), \mathcal{L}^{\vee}, T(-\log )$ (twelve objects).

Here $\mathbb{P}^{1} \times \mathbb{P}^{1} \subset \bar{M}_{0,6}$ are boundary divisors of type $(3,3)$, and $\mathcal{L}$ is a pull-back of the symmetric GIT polarization (the Segre cubic).

We apply this approach to the Losev-Manin moduli space [LM00]. For an $n$-element set $N$, we let $\tilde{N}=\{0, \infty\} \sqcup N$. We write $\overline{L M}_{N}$ for the moduli space of nodal linear chains of projective lines $\mathbb{P}^{1}$ marked by $\tilde{N}$ with 0 on the left tail and $\infty$ on the right tail of the chain. This is a "simplified" version of $\bar{M}_{0, n}$, with linear chains replacing arbitrary trees. The stability conditions are as follows:

- Marked points are never at the nodes.

- Only points marked by $N$ are allowed to coincide with each other.

- Every $\mathbb{P}^{1}$ has at least three special points (marked points or nodes).

The space $\overline{L M}_{N}$ has an action by the group $S_{2} \times S_{N}$ permuting markings. The action of $S_{2}$, which we call the Cremona action, interchanges $\infty$ and 0 . Both $\psi$-classes $\psi_{0}$ and $\psi_{\infty}$ induce birational morphisms $\overline{L M}_{N} \rightarrow \mathbb{P}^{n-1}$, "Kapranov models", which realize $\overline{L M}_{N}$ as an iterated blow-up of $\mathbb{P}^{n-1}$ in $n$ points (standard basis vectors) followed by blowing up $\left(\begin{array}{l}n \\ 2\end{array}\right)$ proper transforms of lines connecting points, etc. (We note that the other $\psi$-classes of $\overline{L M}_{N}$ are trivial.) In these coordinates, the Cremona action is given by the standard Cremona involution

$$
\left(x_{1}: \cdots: x_{n}\right) \rightarrow\left(\frac{1}{x_{1}}: \cdots: \frac{1}{x_{n}}\right) .
$$

The Losev-Manin space $\overline{L M}_{N}$ is a toric variety of dimension $n-1$. Its toric orbits (or their closures, the boundary strata of the moduli space) can be described as follows. Every nontrivial bipartition $N=N_{1} \sqcup N_{2}$ corresponds to the boundary divisor, which we denote by $\delta_{N_{1}}$, parametrizing (degenerations of) chains of two lines $\mathbb{P}^{1}$, one with markings $N_{1} \cup\{0\}$ and another with markings $N_{2} \cup\{\infty\}$. This notation is different from the standard notation for $\bar{M}_{0, n}$ (where an analogous divisor is denoted by $\delta_{N_{1} \cup\{0\}}$ ) but more convenient for us. More generally, every partition $N=N_{1} \sqcup \cdots \sqcup N_{k}$ with $\left|N_{i}\right|>0$ for every $i$ corresponds to the boundary stratum

$$
Z_{N_{1}, \ldots, N_{k}}=\delta_{N_{1}} \cap \delta_{N_{1} \cup N_{2}} \cap \cdots \cap \delta_{N_{1} \cup \cdots \cup N_{k-1}},
$$

which parametrizes (degenerations of) linear chains of lines $\mathbb{P}^{1}$ with points marked by, respectively, $N_{1} \cup\{0\}, N_{2}, \ldots, N_{k-1}, N_{k} \cup\{\infty\}$. We can identify

$$
Z_{N_{1}, \ldots, N_{k}} \simeq \overline{L M}_{N_{1}} \times \cdots \times \overline{L M}_{N_{k}},
$$

where the left node of every $\mathbb{P}^{1}$ is marked by 0 and the right node by $\infty$.

We have a collection of forgetful maps

$$
\pi_{K}: \overline{L M}_{N} \rightarrow \overline{L M}_{N \backslash K}
$$

for every subset $K \subset N$ with $1 \leqslant|K| \leqslant n-1$. The map $\pi_{K}$ is given by forgetting points marked by $K$ and stabilizing. In particular, we can define the cuspidal block $D_{\text {cusp }}^{b}\left(\overline{L M}_{N}\right)$, and applying Theorem 3.5, we show that we have a similar statement as for $\bar{M}_{0, n}$ (Proposition 1.6). 


\section{A.-M. Castravet and J. Tevelev}

Proposition 1.8. The derived category $D^{b}\left(\overline{L M}_{N}\right)$ admits the semi-orthogonal decomposition

$$
D^{b}\left(\overline{L M}_{N}\right)=\left\langle D_{\text {cusp }}^{b}\left(\overline{L M}_{N}\right),\left\{\pi_{K}^{*} D_{\text {cusp }}^{b}\left(\overline{L M}_{N \backslash K}\right)\right\}_{K \subset N}, \mathcal{O}\right\rangle,
$$

where subsets $K$ with $1 \leqslant|K| \leqslant n-2$ are ordered by increasing cardinality.

Next we construct a collection $\hat{\mathbb{G}}$ of sheaves on $\overline{L M}_{N}$. We note that in this definition, and in the rest of the paper, we do not always distinguish notationally between divisors and line bundles.

Definition 1.9. Let $\mathbb{G}_{N}=\left\{G_{1}^{\vee}, \ldots, G_{n-1}^{\vee}\right\}$ be the set of the following line bundles on $\overline{L M}_{N}$ :

$$
G_{a}=a \psi_{0} \otimes \mathcal{O}\left(-(a-1) \sum_{k \in N} \delta_{k}-(a-2) \sum_{k, l \in N} \delta_{k l}-\cdots-\sum_{J \subset N,|J|=a-1} \delta_{J}\right)
$$

for every $a=1, \ldots, n-1$. Let $\hat{\mathbb{G}}$ be the collection of sheaves

$$
\hat{\mathbb{G}}=\bigcup_{Z}\left(i_{Z}\right)_{*}\left[\mathbb{G}_{N_{1}}^{\vee} \otimes \cdots \otimes \mathbb{G}_{N_{t}}^{\vee}\right]
$$

on $\overline{L M}_{N}$ of the form

$$
\mathcal{T}=\left(i_{Z}\right)_{*} \mathcal{L}, \quad \mathcal{L}=G_{a_{1}}^{\vee} \otimes \cdots \otimes G_{a_{t}}^{\vee}
$$

for all strata $Z=Z_{N_{1}, \ldots, N_{t}}$ with $N_{i} \geqslant 2$ for every $i$ and for all $1 \leqslant a_{i} \leqslant\left|N_{i}\right|-1$. Here $i_{Z}: Z \hookrightarrow \overline{L M}_{N}$ is the inclusion map. If $t=1$, we get line bundles $\mathbb{G}_{N}$, and for $t \geqslant 2$, these sheaves are torsion sheaves.

TheOREM 1.10. The set $\hat{\mathbb{G}}$ is a full exceptional collection in $D_{\text {cusp }}^{b}\left(\overline{L M}_{N}\right)$ and is equivariant under the group $S_{2} \times S_{N}$. The number of objects in $\hat{\mathbb{G}}$ is equal to ! $n$, the number of derangements of $n$ objects (permutation without fixed points).

This is our main theorem; its proof occupies Sections 4 and 5. It gives a new curious formula for the number of derangements: ${ }^{2}$

$$
\sum_{\substack{k_{1}+\cdots+k_{t}=n \\
k_{1}, \ldots, k_{t} \geqslant 2}}\left(\begin{array}{ccc}
n \\
k_{1} & \ldots & k_{t}
\end{array}\right)\left(k_{1}-1\right) \cdots\left(k_{t}-1\right)=! n, \quad \text { where }\left(\begin{array}{c}
n \\
k_{1} \ldots
\end{array}\right)=\frac{n !}{k_{1} ! \cdots k_{t} !} .
$$

As a corollary, we see that K-theory of $\overline{L M}_{N}$ is a permutation representation of $S_{2} \times S_{n}$ in a very concrete way, which should be contrasted with description of its equivariant Euler-Poincaré polynomial as an alternating sum in [BM14].

The ordering of $\hat{\mathbb{G}}$ that turns it into an exceptional collection is quite elaborate and discussed in Section 4. The real difficulty though is to prove fullness, which is done in Section 5. Note that fullness would follow at once if phantom subcategories (admissible subcategories with trivial $K$-group) did not exist on smooth projective toric varieties.

Remark 1.11. The line bundles $G_{1}, \ldots, G_{n-1}$ on $\overline{L M}_{n}$ may appear ad hoc, but in fact they have a very nice description in terms of the (minimal) wonderful compactification $\overline{\mathrm{PGL}_{n}}$ of $\mathrm{PGL}_{n}$ (which contains $\overline{L M}_{n}$ as the closure of the maximal torus). Namely, they are precisely the restrictions of generators of the nef cone of $\overline{\mathrm{PGL}_{n}}$; see Proposition 4.14 for a more precise statement. It would be interesting to relate derived categories of $\overline{\mathrm{PGL}}_{n}$ and $\overline{L M}_{n}$.

${ }^{2}$ We are unaware of a combinatorial "bijective" proof of this identity. 


\section{DERIVED CATEGORY OF MODUli OF POINTED CURVES. I}

It is worth noting that we do not know any smooth projective toric varieties $X$ with an action of a finite group $\Gamma$ normalizing the torus action which do not have a $\Gamma$-equivariant exceptional collection $\left\{E_{i}\right\}$ of maximal possible length (equal to the topological Euler characteristic of $X$ ). Its existence would imply that the $\mathrm{K}$-group $K_{0}(X)$ is a permutation $\Gamma$-module. In the Galois setting (when $X$ is defined over a field which is not algebraically closed and $\Gamma$ is the absolute Galois group), an analogous statement was conjectured by A. S. Merkurjev and I. A. Panin [MP97]. Of course, one may further wonder whether $\left\{E_{i}\right\}$ is in fact full, which is related to the existence or not of phantom categories on $X$, another difficult general open question.

We refer to [CT15, CT13, CT12] for background information on the birational geometry of $\bar{M}_{0, n}$, the Losev-Manin space and other related spaces. We refer to [Huy06] for background on semi-orthogonal decompositions.

\section{An equivariant version of Orlov's blow-up theorem}

Orlov's blow-up theorem Theorem 3.3 is a categorification of the following fact. Let $X$ be a smooth projective variety, and let $Y \subseteq X$ be a smooth subvariety of codimension $l$. Let $\tilde{X}$ be the blow-up of $X$ along $Y$. We have a decomposition of cohomology with integral coefficients; see, for example, [Voi07, Theorem 7.31]

$$
H^{*}(\tilde{X}) \simeq\left[H^{*}(Y) \otimes H^{+}\left(\mathbb{P}^{l-1}\right)\right] \oplus H^{*}(X) .
$$

Now consider the following more general situation. Let $Y_{1}, \ldots, Y_{n} \subseteq X$ be smooth transversal subvarieties of codimension $l$. For any subset $I \subseteq\{1, \ldots, n\}$, we denote the intersection $\cap_{i \in I} Y_{i}$ by $Y_{I}$. In particular, $Y_{\emptyset}=X$. Let $q: \tilde{X} \rightarrow X$ be an iterated blow-up of (proper transforms of) $Y_{1}, \ldots, Y_{n}$. Since the intersection is transversal, blow-ups can be done in any order. The analogue of (2.1) in this situation was worked out in [BM13, Proposition 6.1]:

$$
H^{*}(\tilde{X}) \simeq \bigoplus_{\substack{I \subset\{1, \ldots, n\} \\ I \neq \emptyset}}\left[H^{*}\left(Y_{I}\right) \otimes H^{+}\left(\mathbb{P}^{l-1}\right)^{\otimes|I|}\right] \oplus H^{*}(X),
$$

which we are going to rewrite as

$$
H^{*}(\tilde{X}) \simeq \bigoplus_{I \subset\{1, \ldots, n\}}\left[H^{*}\left(Y_{I}\right) \otimes H^{+}\left(\mathbb{P}^{l-1}\right)^{\otimes|I|}\right] .
$$

The analogue of Theorem 3.3 is also straightforward. We fix the following notation. Let $E_{i}$ be the exceptional divisor over $Y_{i}$ for every $i=1, \ldots, n$. For any subset $I \subseteq\{1, \ldots, n\}$, let

$$
E_{I}=q^{-1}\left(Y_{I}\right)=\cap_{i \in I} E_{i} .
$$

In particular, $E_{\emptyset}=\tilde{X}$. Let $i_{I}: E_{I} \hookrightarrow \tilde{X}$ be the inclusion.

Lemma 2.1. Let $\left\{F_{I}^{\beta}\right\}$ be a (full) exceptional collection in $D^{b}\left(Y_{I}\right)$ for every subset $I \subseteq\{1, \ldots, n\}$. There exists a (full) exceptional collection in $D^{b}(\tilde{X})$ with blocks

$$
B_{I, J}=\left(i_{I}\right)_{*}\left[\left(\left.L q\right|_{E_{I}}\right)^{*}\left(F_{I}^{\beta}\right)\left(\sum_{i=1}^{n} J_{i} E_{i}\right)\right]
$$

for every subset $I \subseteq\{1, \ldots, n\}$ (including the empty set) and for every $n$-tuple of integers $J$ such that $J_{i}=0$ if $i \notin I$ and $1 \leqslant J_{i} \leqslant l-1$ for $i \in I$.

The blocks are ordered in any linear order which respects the following partial order: $B_{I^{1}, J^{1}}$ precedes $B_{I^{2}, J^{2}}$ if $\sum_{i=1}^{n} J_{i}^{1} E_{i} \geqslant \sum_{i=1}^{n} J_{i}^{2} E_{i}$ (as effective divisors). 


\section{A.-M. Castravet and J. Tevelev}

Proof. We argue by induction on $n$, the case $n=1$ being Orlov's theorem. We decompose $q: \tilde{X} \rightarrow X$ as a blow-up $q_{0}: X^{\prime} \rightarrow X$ of $Y_{n}$ and an iterated blow-up $q^{\prime}: \tilde{X} \rightarrow X^{\prime}$ of proper transforms $Y_{1}^{\prime}, \ldots, Y_{n-1}^{\prime}$ of $Y_{1}, \ldots, Y_{n-1}$. By Orlov's theorem, $X^{\prime}$ carries a (full) exceptional collection $E^{\prime \alpha}$, namely

$$
i_{*}^{\prime}\left[\left(\left.q_{0}\right|_{E}\right)^{*}\left(F_{n}^{\beta}\right)((l-1) E)\right], \ldots, i_{*}^{\prime}\left[\left(\left.q_{0}\right|_{E}\right)^{*}\left(F_{n}^{\beta}\right)(E)\right], L q_{0}^{*}\left(F_{\emptyset}^{\beta}\right) .
$$

Here $i^{\prime}: E \hookrightarrow X^{\prime}$ is the exceptional divisor, and $\left.q_{0}\right|_{E}$ is a projective bundle.

More generally, for every subset $I^{\prime} \subseteq\{1, \ldots, n-1\}$, let $Y_{I^{\prime}}^{\prime}=\cap_{i \in I^{\prime}} Y_{i}^{\prime}$ be the proper transform of $Y_{I^{\prime}}$ isomorphic to the blow-up of $Y_{I^{\prime}}$ in $Y_{I^{\prime} \cup\{n\}}$. By Orlov's theorem, $Y_{I^{\prime}}^{\prime}$ carries a (full) exceptional collection $F_{I^{\prime}}^{\prime \beta}$, namely

$$
\left(i_{I^{\prime}}^{\prime}\right)_{*}\left[\left(\left.q_{0}\right|_{E_{n}^{I^{\prime}}}\right)^{*}\left(F_{I^{\prime} \cup\{n\}}^{\beta}\right)((l-1) E)\right], \ldots,\left(i_{I^{\prime}}^{\prime}\right)_{*}\left[\left(\left.q_{0}\right|_{E_{n}^{I^{\prime}}}\right)^{*}\left(F_{I^{\prime} \cup\{n\}}^{\beta}\right)(E)\right], L\left(\left.q_{0}\right|_{Y_{I^{\prime}}^{\prime}}\right)^{*}\left(F_{I^{\prime}}^{\beta}\right) .
$$

Here $i_{I^{\prime}}^{\prime}: E_{n}^{I^{\prime}} \hookrightarrow Y_{I^{\prime}}^{\prime}$ is the exceptional divisor over $Y_{I^{\prime} \cup\{n\}}$.

Applying the inductive assumption gives an exceptional collection on $\tilde{X}$ with blocks

$$
\left(i_{I^{\prime}}\right)_{*}\left[\left(\left.L q^{\prime}\right|_{E_{I^{\prime}}}\right)^{*}\left(F_{I^{\prime}}^{\prime \beta}\right)\left(\sum_{i=1}^{n-1} J_{i} E_{i}\right)\right]
$$

for every subset $I^{\prime} \subseteq\{1, \ldots, n-1\}$ (including the empty set) and for every $(n-1)$-tuple of integers $J$ such that $J_{i}=0$ if $i \notin I^{\prime}$ and $1 \leqslant J_{i} \leqslant l-1$ for $i \in I^{\prime}$.

The blocks are ordered in any linear order which respects the following partial order: $B_{I^{\prime 1}, J^{1}}$ precedes $B_{I^{\prime 2}, J^{2}}$ if $\sum_{i=1}^{n-1} J_{i}^{1} E_{i} \geqslant \sum_{i=1}^{n-1} J_{i}^{2} E_{i}$ (as effective divisors). We have to check that these blocks are the same as in the statement of the lemma. It is clear that

$$
\left(\left.L q^{\prime}\right|_{E_{I^{\prime}}}\right)^{*}\left(L\left(\left.q_{0}\right|_{Y_{I^{\prime}}^{\prime}}\right)^{*}\left(F_{I^{\prime}}^{\beta}\right)\right) \simeq\left(\left.L q\right|_{E_{I^{\prime}}}\right)^{*}\left(F_{I^{\prime}}^{\beta}\right) .
$$

This takes care of the last element in $F_{I^{\prime}}^{\prime \beta}$. For the rest, we have to show that

$\left(i_{I^{\prime}}\right)_{*}\left[\left(\left.L q^{\prime}\right|_{E_{I^{\prime}}}\right)^{*}\left(\left(i_{I^{\prime}}^{\prime}\right)_{*}\left[\left(\left.q_{0}\right|_{E_{n}^{I^{\prime}}}\right)^{*}\left(F_{I}^{\beta}\right)\left(J_{n} E\right)\right]\right)\left(\sum_{i=1}^{n-1} J_{i} E_{i}\right)\right] \simeq\left(i_{I}\right)_{*}\left[\left(\left.L q\right|_{E_{I}}\right)^{*}\left(F_{I}^{\beta}\right)\left(\sum_{i=1}^{n} J_{i} E_{i}\right)\right]$,

where $I=I^{\prime} \cup\{n\}$. It suffices to show that

$$
\left(\left.L q^{\prime}\right|_{E_{I^{\prime}}}\right)^{*}\left(\left(i_{I^{\prime}}^{\prime}\right)_{*}\left[\left(\left.q_{0}\right|_{E_{n}^{I^{\prime}}}\right)^{*}\left(F_{I}^{\beta}\right)\left(J_{n} E\right)\right]\right)\left(\sum_{i=1}^{n-1} J_{i} E_{i}\right) \simeq(\phi)_{*}\left[\left(\left.L q\right|_{E_{I}}\right)^{*}\left(F_{I}^{\beta}\right)\left(\sum_{i=1}^{n} J_{i} E_{i}\right)\right],
$$

where $\phi: E_{I} \hookrightarrow E_{I^{\prime}}$ is the inclusion. Applying the projection formula, we reduce this to

$$
\left(\left.L q^{\prime}\right|_{E_{I^{\prime}}}\right)^{*}\left(\left(i_{I^{\prime}}^{\prime}\right)_{*}\left[\left(\left.q_{0}\right|_{E_{n}^{I^{\prime}}}\right)^{*}\left(F_{I}^{\beta}\right)\right]\right) \simeq(\phi)_{*}\left[\left(\left.L q\right|_{E_{I}}\right)^{*}\left(F_{I}^{\beta}\right)\right]
$$

which follows by flat base change.

The last order of business is to prove the claim about the order of the blocks. We made a choice of blowing up $Y_{n}$ first; accordingly, the collection has blocks $B_{I^{\prime}, J^{\prime}}$ for every subset $I^{\prime} \subseteq\{1, \ldots, n-1\}$ (including the empty set) and for every $(n-1)$-tuple of integers $J^{\prime}$ such that $J_{i}^{\prime}=0$ if $i \notin I^{\prime}$ and $1 \leqslant J_{i}^{\prime} \leqslant l-1$ for $i \in I^{\prime}$. The blocks are ordered in any linear order which respects the following partial order: $B_{I^{\prime 1}, J^{\prime 1}} \prec B_{I^{\prime 2}, J^{\prime 2}}$ if $\sum_{i=1}^{n-1} J_{i}^{\prime 1} E_{i}>\sum_{i=1}^{n-1} J_{i}^{\prime 2} E_{i}$ (as effective divisors). Each block $B_{I^{\prime}, J^{\prime}}$ is a sequence of blocks $B_{I, J}$ from the statement of the lemma, where $I \cap\{1, \ldots, n-1\}=I^{\prime}$ and $J_{i}=J_{i}^{\prime}$ for $i<n$. They are ordered in the decreasing order by $J_{n}$. In particular, if $B_{I^{1}, J^{1}}$ precedes $B_{I^{2}, J^{2}}$, then either $\sum_{i=1}^{n} J_{i}^{1} E_{i}-\sum_{i=1}^{n} J_{i}^{2} E_{i}$ is an effective divisor, or $\sum_{i=1}^{n-1} J_{i}^{2} E_{i}-\sum_{i=1}^{n-1} J_{i}^{1} E_{i}$ is not effective. Therefore, it suffices to prove that for any two blocks 


\section{DERIVED CATEGORY OF MODUli OF POINTED CURVES. I}

$B_{I^{1}, J^{1}}$ and $B_{I^{2}, J^{2}}$, if $\sum_{i=1}^{n} J_{i}^{1} E_{i}-\sum_{i=1}^{n} J_{i}^{2} E_{i}$ is not an effective divisor, then $\left\{B_{I^{1}, J^{1}}, B_{I^{2}, J^{2}}\right\}$ is an exceptional sequence. If $\sum_{i=1}^{n-1} J_{i}^{1} E_{i}-\sum_{i=1}^{n-1} J_{i}^{2} E_{i}$ is not effective, then we are done by the above. But if it is effective, then $\sum_{i=2}^{n} J_{i}^{1} E_{i}-\sum_{i=2}^{n} J_{i}^{2} E_{i}$ is not effective, and we are again done by the above (by changing the order of blow-ups and blowing up $Y_{1}$ first).

Remark 2.2. The same argument shows, more generally, that even in the absence of exceptional collections, there exists a semi-orthogonal decomposition of $D^{b}(\tilde{X})$ with blocks

$$
B_{I, J}=\left(i_{I}\right)_{*}\left[\left(\left.L q\right|_{E_{I}}\right)^{*}\left(D^{b}\left(Y_{I}\right)\right)\left(\sum_{i=1}^{n} J_{i} E_{i}\right)\right]
$$

(with the same notation and order as in the lemma). We stated the lemma for exceptional collections with an eye toward its equivariant version.

Continuing with the set-up of Lemma 2.1, let $G$ be a finite group acting on $X$ permuting $Y_{1}, \ldots, Y_{n}$. Then it also acts on $\tilde{X}$, and the morphism $q$ is $G$-equivariant. Let $G_{I} \subseteq G$ be the normalizer of $Y_{I}$ for each subset $I \subseteq\{1, \ldots, n\}$ (in particular, $G_{\emptyset}=G$ ).

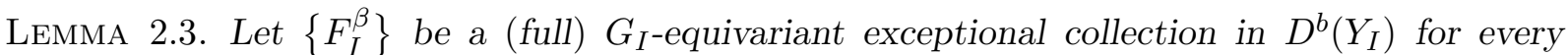
subset $I \subseteq\{1, \ldots, n\}$. We assume that if $Y_{I}=g Y_{I^{\prime}}$ for some $g \in G$, then $\left\{F_{I}^{\beta}\right\}=g\left\{F_{I^{\prime}}^{\beta}\right\}$. There exists a (full) $G$-equivariant exceptional collection in $D^{b}(\tilde{X})$ with blocks $B_{I, J}$ (the same as in Lemma 2.1).

Proof. It suffices to observe that the blocks $B_{I, J}$ are permuted by $G$.

Next we recall a few facts and notation from [BM13] in order to prove Theorem 1.2. The subgroup $S_{k} \times S_{n-k} \subseteq S_{n}$ preserves the weight a and therefore acts on $\bar{M}_{k, l}^{n}$. We have $\left(S_{k} \times S_{n-k}\right)$ equivariant reduction morphisms

$$
\bar{M}_{k, 1}^{n} \rightarrow \bar{M}_{k, 2}^{n} \rightarrow \cdots \rightarrow \bar{M}_{k, r(n, k)}^{n},
$$

where the first map is an isomorphism. Each of the maps in (2.3) is an iterated blow-up of transversal loci of the same codimension permuted by $S_{k} \times S_{n-k}$. Specifically, for every subset $I \subset\{k+1, \ldots, n\}$ of cardinality $l+1$, let $\bar{M}_{k, l+1}^{n}(I) \subseteq \bar{M}_{k, l+1}^{n}$ be the closure of the locus where points marked by $I$ collide. The reduction morphism $\bar{M}_{k, l}^{n} \rightarrow \bar{M}_{k, l+1}^{n}$ is the blow-up along the transversal union $\cup_{I} \bar{M}_{k, l+1}^{n}(I)$ of subvarieties of codimension $l$, where $I$ runs over all subsets of $\{k+1, \ldots, n\}$ of cardinality $l+1$; see [BM13, Lemma 3.1]. Intersections of these loci are described in [BM13, Section 3.2] as follows. Let $I_{1}, \ldots, I_{m} \subset\{k+1, \ldots, n\}$ be subsets of cardinality $l+1$. Then $\cap_{i=1}^{m} \bar{M}_{k, l+1}^{n}\left(I_{i}\right) \neq \emptyset$ if and only if the subsets $I_{1}, \ldots, I_{m}$ are disjoint. In this case, the intersection is isomorphic to $\bar{M}_{k+m, l+1}^{n-l m}$. Moreover, the stabilizer of this stratum in $S_{k} \times S_{n-k}$ acts on it through a subquotient contained in $S_{k+m, n-l m-k-m}$. Applying Lemma 2.3 proves Theorem 1.2.

\section{The cuspidal block}

Recall that by Definition 1.4, we call an object $E \in D^{b}(X)$ cuspidal with respect to a given collection of morphisms $\pi_{i}: X \rightarrow X_{i}$ (for $i \in I$ ) between smooth projective varieties if

$$
R \pi_{i *} E=0 \quad \text { for every } i \in I .
$$

The cuspidal block is the full triangulated subcategory of cuspidal objects $D_{\text {cusp }}^{b}(X) \subset D^{b}(X)$. 


\section{A.-M. Castravet and J. Tevelev}

Lemma-Definition 3.1. In the set-up of Definition 1.4, the support of any cuspidal object is a union of irreducible closed subsets $Z \subset X$ such that

$$
\operatorname{dim} \pi_{i}(Z)<\operatorname{dim} Z \quad \text { for every } i \in S .
$$

We call any subset $Z$ with this property (independently of whether they are the support of a cuspidal object or not) massive. Recall that the topological support of an object $E \in D^{b}(X)$ is the support of its cohomology sheaves.

Proof. Let $Z$ be the topological support of $E \in D^{b}(X)$. Suppose that $Z$ contains an irreducible component $Z_{0}$ such that $\operatorname{dim} \pi_{i}\left(Z_{0}\right)=\operatorname{dim} Z_{0}$. We write $\pi:=\pi_{i}$ and $Y:=X_{i}$ as we will not need other maps and spaces. By passing to an open subset of $Y$ and taking its preimage under $\pi$, we can assume that $Z$ is a disjoint union of $Z_{0}$ and $Z_{1}$ (with $Z_{1}$ possibly empty and not necessarily irreducible). We may also assume that $\left.\pi\right|_{Z_{0}}$ is finite. It is well known [Orl11, Section 2] that by changing $E$ to an isomorphic object, we may assume that $E$ is a bounded complex of sheaves supported on $Z$. Thus $E=R i_{*} \tilde{E}$, where $i: \tilde{Z} \hookrightarrow X$ is an infinitesimal thickening of $Z$ and $\tilde{E} \in D^{b}(\tilde{Z})$. Note that $\tilde{Z}$ is a disjoint union of subschemes $\tilde{Z}_{0}$ and $\tilde{Z}_{1}$ (with reduced subschemes $Z_{0}$ and $Z_{1}$ ). In particular, $\tilde{E}=\tilde{E}_{0} \oplus \tilde{E}_{1}$, where $\tilde{E}_{0}$ and $\tilde{E}_{1}$ are pull-backs of $\tilde{E}$ to $Z_{0}$ and $Z_{1}$, respectively. It follows that $R \tilde{\pi}_{*}\left(\tilde{E}_{0}\right)=0$, where $\tilde{\pi}=\pi \circ i$. Since $\tilde{E}_{0} \neq 0$ and the map $\tilde{\pi}$ is affine, this gives a contradiction. Indeed, if $\pi: X \rightarrow Y$ is an affine morphism of schemes, then $R \pi_{*} E=0$ for some $E \in D_{Q \operatorname{Coh}}\left(\mathcal{O}_{X}\right)$ implies that $E=0$; see [Sta20, Tag 0AVV].

We refer to the survey [Kuz16] for definitions and basic facts concerning semi-orthogonal decompositions in algebraic geometry. The following is well known; see, for example, [Kuz08, Lemma 2.4].

Proposition 3.2. Let $\pi: X \rightarrow Y$ be a morphism of smooth projective varieties such that $R \pi_{*} \mathcal{O}_{X}=\mathcal{O}_{Y}$. Then $L \pi^{*} D^{b}(Y)$ is an admissible subcategory of $D^{b}(X)$, and there is a semiorthogonal decomposition

$$
D^{b}(X)=\left\langle D_{\text {cusp }}^{b}(X), L \pi^{*} D^{b}(Y)\right\rangle .
$$

In particular, $D_{\text {cusp }}^{b}(X)$ is an admissible subcategory.

Classical situations of this sort are provided by Orlov's theorems [Or193] on derived categories of a projective bundle and of a blow-up, which can be reformulated as follows.

Theorem 3.3. Let $\pi: X \rightarrow Y$ be a projective bundle of rank $r$, with $Y$ a smooth projective variety. Then $D_{\text {cusp }}^{b}(X)$ is an admissible subcategory of $D^{b}(X)$ and $D_{\text {cusp }}^{b}(X)$ has a semi-orthogonal decomposition

$$
\left\langle\pi^{*} D^{b}(Y) \otimes \mathcal{O}_{\pi}(-r), \ldots, \pi^{*} D^{b}(Y) \otimes \mathcal{O}_{\pi}(-1)\right\rangle .
$$

Theorem 3.4. Let $p: X \rightarrow Y$ be a blow-up of a smooth subvariety $Z$ of codimension $r+1$ of a smooth projective variety $Y$. Let $i: E \rightarrow X$ be the exceptional divisor, and let $\pi=\left.p\right|_{Z}$. Then $D_{\text {cusp }}^{b}(X)$ is an admissible subcategory of $D^{b}(X)$ and has a semi-orthogonal decomposition

$$
\left\langle R i_{*}\left[\pi^{*} D^{b}(Z) \otimes \mathcal{O}_{\pi}(-r)\right], \ldots, R i_{*}\left[\pi^{*} D^{b}(Z) \otimes \mathcal{O}_{\pi}(-1)\right]\right\rangle .
$$

In order to generalize Proposition 3.2 to the set-up of several morphisms, we impose compatibility conditions. In subsequent sections, we will consider several variants of moduli spaces of rational pointed curves, which will all fit into this framework. 


\section{DERIVED CATEGORY OF MODUli OF POINTED CURVES. I}

TheOREm 3.5. Let $\mathbb{N}$ be the category of finite subsets of a fixed set with inclusions as morphisms. Let $X$ be a contravariant functor from $\mathbb{N}$ to the category of smooth projective varieties. For every $T \subseteq S$, we refer to the morphism $X_{S} \rightarrow X_{T}$ as the forgetful map and denote it by $\pi_{S \backslash T}$. We impose three assumptions:

(1) We have

$$
R \pi_{i *} \mathcal{O}_{X_{S}}=\mathcal{O}_{X_{S \backslash\{i\}}} \quad \text { for every } i \in S .
$$

(2) For all $i, k \in S, i \neq k$, the morphisms

$$
\pi_{i}: X_{S \backslash\{k\}} \rightarrow X_{S \backslash\{i, k\}}, \quad \pi_{k}: X_{S \backslash\{i\}} \rightarrow X_{S \backslash\{i, k\}} \quad \text { are Tor-independent }
$$

(as defined in [Sta20, Definition 36.21.2]).

(3) If we let

$$
Y:=X_{S \backslash\{i\}} \times_{X_{S \backslash\{i, k\}}} X_{S \backslash\{k\}}
$$

and $\alpha_{i, k}: X_{S} \rightarrow Y$ is the map induced by $\pi_{i}$ and $\pi_{k}$, we have

$$
R \alpha_{i, k_{*}} \mathcal{O}_{X_{S}}=\mathcal{O}_{Y}
$$

Under these assumptions, we have a semi-orthogonal decomposition (s.o.d.)

$$
D^{b}\left(X_{S}\right)=\left\langle D_{\text {cusp }}^{b}\left(X_{S}\right),\left\{L \pi_{K}^{*} D_{\text {cusp }}^{b}\left(X_{S \backslash K}\right)\right\}_{K \subset S}, L \pi_{S}^{*} D^{b}\left(X_{\emptyset}\right)\right\rangle,
$$

where $K$ runs over proper subsets of $S$ in order of increasing cardinality. In particular, $D_{\text {cusp }}^{b}\left(X_{S}\right)$ is an admissible subcategory of $D^{b}\left(X_{S}\right)$.

Following a suggestion of A. Kuznetsov, we start with an abstract "inclusion-exclusion" principle in triangulated categories. Perhaps we should remark that semi-orthogonal decompositions do not intersect well in general, as a simple example of $D^{b}\left(\mathbb{P}^{1}\right)=\langle\mathcal{O}, \mathcal{O}(1)\rangle=\langle\mathcal{O}(2), \mathcal{O}(3)\rangle$ shows. However, we have the following.

Lemma 3.6. Let $\mathcal{T}$ be a triangulated category with several semi-orthogonal decompositions

$$
\mathcal{T}=\left\langle A_{1}, B_{1}\right\rangle=\left\langle A_{2}, B_{2}\right\rangle=\cdots=\left\langle A_{n}, B_{n}\right\rangle .
$$

Suppose that the projection functors $\beta_{i}: \mathcal{T} \rightarrow B_{i}$ (in the $i$ th decomposition) have the property that for every $j$,

$$
\beta_{i}\left(A_{j}\right) \subset A_{j}, \quad \beta_{i}\left(B_{j}\right) \subset B_{j}
$$

Then we have a s.o.d.

$$
\mathcal{T}=\left\langle\mathcal{T}_{K}\right\rangle_{K}, \quad \text { where } \quad \mathcal{T}_{K}=\left(\cap_{i \notin K} A_{i}\right) \cap\left(\cap_{i \in K} B_{i}\right)
$$

and $K$ runs over subsets of $\{1, \ldots, n\}$ in the order of increasing cardinality. In particular, $\mathcal{T}_{\emptyset}=$ $A_{1} \cap \cdots \cap A_{n}$ is an admissible subcategory of $\mathcal{T}$.

Proof. For all subsets $T \subseteq S:=\{1, \ldots, n\}$, we consider a full triangulated subcategory $A_{T}=$ $\cap_{i \in T} A_{i}$. We prove, more generally, that there is a semi-orthogonal decomposition $A_{T}=\left\langle\mathcal{T}_{K}\right\rangle$, where $K$ runs over subsets of $S$ containing $T$ in order of increasing cardinality. The case $T=\emptyset$ is the statement in the theorem.

We argue by induction on $n=|S|$ and by downwards induction on $|T|$ for a fixed $n$. If $n=1$ or $T=S$, then there is nothing to prove. Let $i \in S \backslash T$. Without loss of generality, we assume $i=1$. 


\section{A.-M. Castravet and J. Tevelev}

We claim that the semi-orthogonal decomposition $\mathcal{T}=\left\langle A_{1}, B_{1}\right\rangle$ induces a semi-orthogonal decomposition

$$
A_{T}=\left\langle A_{T} \cap A_{1}, A_{T} \cap B_{1}\right\rangle .
$$

Indeed, the semi-orthogonality is obvious, and, moreover, every object $X$ in $A_{T}$ fits into a distinguished triangle $\beta_{1}(X) \rightarrow X \rightarrow Y \rightarrow$ with $Y \in A_{1}$. Since $\beta_{1}$ preserves $A_{T}$ by our assumptions, $\beta_{1}(X) \in A_{T} \cap B_{1}$. It follows that $Y \in A_{T}$ as well.

By the induction assumption, we have semi-orthogonal decompositions

$$
A_{T} \cap A_{1}=A_{T \cup\{1\}}=\left\langle\mathcal{T}_{K}\right\rangle \text { and } A_{T}=\left\langle\mathcal{T}_{K^{\prime}}^{\prime}\right\rangle,
$$

where $K$ runs over subsets of $S$ containing $T \cup\{1\}$ and $K^{\prime}$ over subsets of $S \backslash\{1\}$ containing $T$ and

$$
\mathcal{T}_{K^{\prime}}^{\prime}=\left(\cap_{i \notin K \cup\{1\}} A_{i}\right) \cap\left(\cap_{i \in K} B_{i}\right) .
$$

We claim that the semi-orthogonal decomposition $A_{T}=\left\langle\mathcal{T}_{K^{\prime}}^{\prime}\right\rangle$ induces the semi-orthogonal decomposition

$$
A_{T} \cap B_{1}=\left\langle\mathcal{T}_{K^{\prime}}^{\prime} \cap B_{1}\right\rangle=\left\langle\mathcal{T}_{K^{\prime} \cup\{1\}}\right\rangle .
$$

Indeed, the semi-orthogonality is clear. By the definition of the semi-orthogonal decomposition, for every object $X \in A_{T} \cap B_{1}$, we can write a sequence of morphisms ("filtration")

$$
0 \rightarrow \cdots \rightarrow T_{K_{1}^{\prime}} \rightarrow T_{K_{2}^{\prime}} \rightarrow \cdots \rightarrow X \rightarrow 0
$$

such that every morphism is included in the distinguished triangle

$$
T_{K_{1}^{\prime}} \rightarrow T_{K_{2}^{\prime}} \rightarrow X_{K_{1}^{\prime}} \rightarrow
$$

with $X_{K_{1}^{\prime}} \in \mathcal{T}_{K_{1}^{\prime}}^{\prime}$. Applying the functor $\beta_{1}$ to this sequence and using our assumptions gives a filtration of $X$ with subquotients $\beta_{1}\left(X_{K_{1}^{\prime}}\right) \in \mathcal{T}_{K_{1}^{\prime}}^{\prime} \cap B_{1}$.

Combining these observations with (3.4), we get a semi-orthogonal decomposition

$$
A_{T}=\left\langle\mathcal{T}_{K}, \mathcal{T}_{K^{\prime} \cup\{1\}}\right\rangle,
$$

where $K$ runs over subsets of $S$ containing $T \cup\{1\}$ and $K^{\prime}$ runs over subsets of $S \backslash\{1\}$ containing $T$, both in order of increasing cardinality.

Finally, we have to show that we can reorder blocks to put them in the order of increasing cardinality. If $\left|K_{1}\right|<\left|K_{2}\right|$, then choose an index $j \in K_{2} \backslash K_{1}$. Then $\mathcal{T}_{K_{1}} \subset A_{j}$ and $\mathcal{T}_{K_{2}} \subset B_{j}$. Thus $\mathcal{T}_{K_{1}} \subset \mathcal{T}_{K_{2}}^{\perp}$.

Proof of Theorem 3.5. By Proposition 3.2, we have s.o.d.'s $D^{b}\left(X_{S}\right)=\left\langle A_{i}, B_{i}\right\rangle$, where

$$
A_{i}=\left\{E \in D^{b}\left(X_{S}\right) \mid R \pi_{i *} E=0\right\} \quad \text { and } \quad B_{i}=L \pi_{i}^{*}\left(D^{b}\left(X_{S \backslash\{i\}}\right)\right) .
$$

We now apply Lemma 3.6 to $\left\langle A_{i}, B_{i}\right\rangle$. The projection operators are

$$
\beta_{i}=L \pi_{i}^{*} R \pi_{i *} .
$$

Note that for all $i, k \in S$ with $i \neq k$ and all $E \in D^{b}\left(X_{S \backslash\{k\}}\right)$, we have

$$
R \pi_{i *} L \pi_{k}{ }^{*} E \simeq L \pi_{k}{ }^{*} R \pi_{i *} E
$$

since, by assumption, $\pi_{i}$ and $\pi_{k}$ are Tor-independent. This follows from assumption (2) combined with cohomology and base change: if $\pi_{i}^{\prime}$ and $\pi_{k}^{\prime}$ are the projection maps from $Y=X_{S \backslash\{i\}} \times_{X_{S \backslash\{i, k\}}}$ $X_{S \backslash\{k\}}$ and $\alpha: X_{S} \rightarrow Y$ is the canonical map, we have

$$
R \pi_{i *} L \pi_{k}^{*} E=R \pi_{i *}^{\prime} R \alpha_{*} L \alpha^{*} L \pi_{k}^{\prime *} E=R \pi_{i *}^{\prime} L \pi_{k}^{\prime *} E=L \pi_{k}^{*} R \pi_{i *} E
$$




\section{DERIVED CATEGORY OF MODULI OF POINTED CURVES. I}

where the second equality is by the projection formula and (3.3). It follows that

$$
R \pi_{j *} L \pi_{i}^{*} R \pi_{i *}=L \pi_{i}^{*} R \pi_{j *} R \pi_{i *}=L \pi_{i}^{*} R \pi_{i *} R \pi_{j *},
$$

and in particular $\beta_{i}\left(A_{j}\right) \subset A_{j}$. Also,

$$
L \pi_{i}^{*} R \pi_{i *} L \pi_{j}^{*}=L \pi_{i}^{*} L \pi_{j}^{*} R \pi_{i *}=L \pi_{j}^{*} L \pi_{i}^{*} R \pi_{i *},
$$

and thus $\beta_{i}\left(B_{j}\right) \subset B_{j}$.

It remains to show that, in the notation of Lemma 3.6, we have $D^{b}\left(X_{S}\right)_{K}=L \pi_{K}^{*} D_{\text {cusp }}^{b}\left(X_{S \backslash K}\right)$ for every subset $K \subset T$. Equivalently,

$$
\bigcap_{i \in K} B_{i}=L \pi_{K}^{*} D^{b}\left(X_{S \backslash K}\right) .
$$

We can assume that $K=\{1, \ldots, k\}$. Then it follows from (3.5) that $\beta_{1} \circ \cdots \circ \beta_{k}=L \pi_{K}^{*} R \pi_{K *}$. Thus every object from the left-hand side of (3.6) is isomorphic to an object from the right-hand side, and vice versa.

Example 3.7. Let $X_{S}=\left(\mathbb{P}^{1}\right)^{S}$ with projections as forgetful maps. Conditions (1), (2) and (3) of Theorem 3.5 are clearly satisfied. The subset $X_{S}$ is the only massive one. Applying Theorem 3.3 repeatedly, it follows that

$$
D_{\text {cusp }}^{b}\left(X_{S}\right)=\langle\mathcal{O}(-1,-1, \ldots,-1)\rangle ;
$$

that is, every object in $D_{\text {cusp }}^{b}\left(X_{S}\right)$ is isomorphic to $\mathcal{O}(-1,-1, \ldots,-1) \otimes_{k} K$, where $K$ is a complex of vector spaces. Moreover, the semi-orthogonal decomposition of Theorem 3.5 is induced by a standard exceptional collection of $2^{|S|}$ line bundles $\mathcal{O}\left(n_{1}, \ldots, n_{|S|}\right)$, where $n_{i}=0$ or -1 for every $i$.

Note that this collection is obviously equivariant under the action of $\operatorname{Aut}\left(X_{S}\right)$, which is the semidirect product of $S_{n}$ and $\left(\mathrm{PGL}_{2}\right)^{n}$ for $n=|S|$. Various moduli spaces considered in this paper can be viewed as "compactified quotients" of this basic example modulo $\mathbb{G}_{m}$ or $\mathrm{PGL}_{2}$.

Proof of Proposition 1.6. Recall that we need to prove that $D^{b}\left(\bar{M}_{N}\right)$ admits a semi-orthogonal decomposition

$$
D^{b}\left(\bar{M}_{N}\right)=\left\langle D_{\text {cusp }}^{b}\left(\bar{M}_{N}\right),\left\{\pi_{K}^{*} D_{\text {cusp }}^{b}\left(\bar{M}_{N \backslash K}\right)\right\}_{K \subset N}, \mathcal{O}\right\rangle
$$

where $K$ runs over subsets with $1 \leqslant|K| \leqslant n-4$ in order of increasing cardinality $|K|$. We apply Theorem 3.5. All conditions (1), (2) and (3) are satisfied. Recall that a simple criterion for Torindependence for maps $X \rightarrow S$ and $T \rightarrow S$ is that one of them is flat. Hence, condition (2) holds as the forgetful maps $\pi_{i}: \bar{M}_{0, n} \rightarrow \bar{M}_{0, n-1}$ are flat. Condition (3) holds as the map is birational and the image has rational singularities [Kee92, Introduction, p. 548].

The following proof is similar.

Proof of Proposition 1.8. Recall that we need to prove that $D^{b}\left(\overline{L M}_{N}\right)$ admits the semi-orthogonal decomposition

$$
D^{b}\left(\overline{L M}_{N}\right)=\left\langle D_{\text {cusp }}^{b}\left(\overline{L M}_{N}\right),\left\{\pi_{K}^{*} D_{\text {cusp }}^{b}\left(\overline{L M}_{N \backslash K}\right)\right\}_{K \subset N}, \mathcal{O}\right\rangle,
$$

where subsets $K$ with $1 \leqslant|K| \leqslant n-2$ are ordered by increasing cardinality. We apply Theorem 3.5 to the forgetful maps

$$
\pi_{i}: \overline{L M}_{N} \rightarrow \overline{L M}_{N \backslash\{i\}} \quad(i \in N) .
$$




\section{A.-M. Castravet and J. Tevelev}

All conditions (1), (2) and (3) are satisfied. Note again that the forgetful maps $\pi_{i}$ for $i \in N$ are flat (they give the universal family); hence, condition (2) holds. Condition (3) holds because $\alpha_{i j}$ is birational and $Y$ has toroidal, and therefore rational, singularities.

\section{The exceptional collection $\hat{\mathbb{G}}$ on the Losev-Manin space}

Proposition 4.1. An irreducible subset $Z \subset \overline{L M}_{N}$ is massive if and only if $Z$ is a boundary stratum of the form $Z_{N_{1}, \ldots, N_{t}}$ with $\left|N_{i}\right| \geqslant 2$ for $i=1, \ldots, t$.

Proof. Let $Z$ be a boundary stratum. If $N_{i}=\{a\}$ for some $i$, then $\pi_{a}$ restricted to $Z$ is oneto-one. Hence $Z$ is not a massive subset. On the other hand, if $\left|N_{i}\right| \geqslant 2$ for every $i$, then $Z$ is a massive subset. It remains to show that if $Z$ is a proper irreducible subset of a boundary stratum which intersects its interior, then $Z$ is not massive. But the interior of any stratum is an algebraic torus $\mathbb{G}_{m}^{r}$, and projections onto coordinate subtori are realizable as forgetful maps. Thus $Z$ cannot be massive.

Proposition 4.2. The ranks of the $K$-groups of $D^{b}\left(\overline{L M}_{n}\right)$ and $D_{\text {cusp }}^{b}\left(\overline{L M}_{n}\right)$ are equal to $n$ ! and $! n$, respectively.

Proof. Since $\overline{L M}_{N}$ is a toric variety, its $K$-group is a free Abelian group and its topological Euler characteristic (and thus the rank of its K-group) is equal to the number of torus fixed points, which are clearly parametrized by permutations of $N$. The second part of the proposition follows because both the rank of the K-group of $D_{\text {cusp }}^{b}\left(\overline{L M}_{n}\right)$ (by Proposition 1.8) and !n (by obvious reasons) satisfy the same recursion

$$
n !=! n+\sum_{1 \leqslant k \leqslant n-1}\left(\begin{array}{l}
n \\
k
\end{array}\right) !(n-k)+1 .
$$

Hence these numbers agree.

Proof of formula (1.3). Recall that formula (1.3) states that

$$
\sum_{\substack{k_{1}+\cdots+k_{t}=n \\
k_{1}, \ldots, k_{t} \geqslant 2}}\left(\begin{array}{ccc}
n \\
k_{1} & \ldots & k_{t}
\end{array}\right)\left(k_{1}-1\right) \cdots\left(k_{t}-1\right)=! n, \quad \text { where }\left(\begin{array}{cc}
n \\
k_{1} \ldots & \ldots
\end{array}\right)=\frac{n !}{k_{1} ! \ldots k_{t} !} .
$$

We denote the left-hand side by $d_{n}$ and set $d_{0}=1$ and $d_{1}=0$. Let

$$
A=\sum_{n \geqslant 2}(n-1) \frac{x^{n}}{n !}=x^{2}\left(\frac{e^{x}-1}{x}\right)^{\prime}=e^{x}(x-1)+1 .
$$

Then we have

$$
\sum_{m \geqslant 0} \frac{d_{m}}{m !} x^{m}=1+A+A^{2}+A^{3}+\cdots=\frac{1}{1-A}=\frac{e^{-x}}{1-x} .
$$

But (4.1) implies that

$$
\frac{1}{1-x}=\left(\sum_{m \geqslant 0} ! m \frac{x^{m}}{m !}\right)\left(\sum_{n \geqslant 0} \frac{x^{n}}{n !}\right)
$$

(where we set $! 0=0 !=1$ ). Hence $d_{n}=! n$, and we are done. 


\section{DERIVED CATEGORY OF MODUli OF POINTED CURVES. I}

Lemma 4.3. (1) Every $G_{a}$ is $S_{N}$-invariant, and Cremona action takes it to $G_{n-a}$.

(2) We have $G_{1}=\psi_{0}$ and $G_{n-1}=\psi_{\infty}$.

(3) For every boundary divisor $\delta=\delta_{N_{1}} \simeq \overline{L M}_{N_{1}} \times \overline{L M}_{N_{2}}$, we have

$$
G_{a \mid \delta}= \begin{cases}G_{a} \otimes \mathcal{O} & \text { if } a<\left|N_{1}\right|, \\ \mathcal{O} & \text { if } a=\left|N_{1}\right|, \\ \mathcal{O} \otimes G_{a-\left|N_{1}\right|} \mid & \text { if } a>\left|N_{1}\right| .\end{cases}
$$

Proof. Direct calculation.

Notation 4.4. For an object $F \in D^{b}(X)$, we often use the notation $R \Gamma(F)$ instead of $R \Gamma(X, F)$ when the space $X$ is clear from the context.

LEMMA 4.5. (1) Every $G_{a}$ is nef (and hence globally generated), of relative degree 1 with respect to any forgetful map $\pi_{i}$, for $i \in N$.

(2) We have $G_{a}^{\vee} \in D_{\text {cusp }}^{b}\left(\overline{L M}_{n}\right)$. In particular, each $G_{a}^{\vee}$ is acyclic.

(3) We have $R \operatorname{Hom}\left(G_{a}, G_{b}\right)=0$ if $a \neq b$.

(4) We have $R \Gamma\left(-\psi_{0}+G_{a}-G_{b}\right)=R \Gamma\left(-\psi_{\infty}+G_{b}-G_{a}\right)=0$ if $a<b$.

In particular, $\mathbb{G}_{N}$ is an $\left(S_{2} \times S_{n}\right)$-equivariant exceptional (in fact pairwise orthogonal) collection of $n-1$ line bundles in $D_{\text {cusp }}^{b}\left(\overline{L M}_{N}\right)$.

Proof. Since $\overline{L M}_{n}$ is a toric variety, part (1) will follow if $G_{a}$ is non-negative on toric boundary curves. This follows from Lemma $4.3(3)$ by induction on the dimension. Since the restriction of $G_{a}^{\vee}$ to each fiber of $\pi_{i}$ has vanishing cohomology, part (2) follows by cohomology and base change. Since $R \operatorname{Hom}\left(G_{a}, G_{b}\right)=R \Gamma\left(-G_{a}+G_{b}\right)$ and we can assume $a>b$ (by applying Cremona action), parts (3) and (4) both follow from Lemma 4.6.

Lemma 4.6. Consider the divisor

$$
D=-d H+\sum m_{I} E_{I}
$$

on $\bar{M}_{0, n}$ or $\overline{L M}_{N}$ written in some Kapranov model. The divisor $D$ is acyclic if

$$
1 \leqslant d \leqslant n-3 \text { and } 0 \leqslant m_{I} \leqslant n-3-\# I .
$$

Proof. By consecutively restricting to boundary divisors $E_{I}$ starting with those with the largest $\# I$ and continuing to those with smaller \#I, we see that all the restrictions are acyclic; hence, $D$ has the same cohomology as $-d H$. Clearly, $-d H$ is acyclic if and only if $1 \leqslant d \leqslant n-3$.

Lemma 4.7. The set $\hat{\mathbb{G}}$ is a collection of !n sheaves in $D_{\text {cusp }}^{b}\left(\overline{L M}_{N}\right)$.

Proof. Follows from (1.3) and Lemma 4.5(2).

It is worth mentioning that if $i: Z \hookrightarrow X$ is a closed embedding of smooth projective varieties and $Z \neq X$, then the functor $R i_{*}: D^{b}(Z) \rightarrow D^{b}(X)$ is in general not fully faithful. Therefore, even though all sheaves in $\hat{\mathbb{G}}$ are clearly exceptional in the derived category of their respective support (being line bundles on a rational variety), we still have to prove the following.

Lemma 4.8. All sheaves in $\hat{\mathbb{G}}$ are exceptional. 


\section{A.-M. Castravet and J. Tevelev}

Proof. All sheaves in $\hat{\mathbb{G}}$ are of the form $i_{*} i^{*} \mathcal{L}=R i_{*} L i^{*} \mathcal{L}$, where $\mathcal{L}$ is an invertible sheaf on $\overline{L M}_{N}$ and $i$ is an embedding of some massive stratum $Z$. We have

$$
\begin{aligned}
R \operatorname{Hom}\left(R i_{*} L i^{*} \mathcal{L}, R i_{*} L i^{*} \mathcal{L}\right) & =R \operatorname{Hom}\left(\mathcal{L} \stackrel{L}{\otimes} R i_{*} \mathcal{O}_{Z}, \mathcal{L} \stackrel{L}{\otimes} R i_{*} \mathcal{O}_{Z}\right) \\
& =R \operatorname{Hom}\left(R i_{*} \mathcal{O}_{Z}, R i_{*} \mathcal{O}_{Z}\right) .
\end{aligned}
$$

So it suffices to prove that $R i_{*} \mathcal{O}_{Z}=i_{*} \mathcal{O}_{Z}$ is an exceptional object. Let $Z$ be the intersection of boundary divisors $D_{1}, \ldots, D_{s}$. Resolving $i_{*} \mathcal{O}_{Z}$ by the Koszul complex

$$
\cdots \rightarrow \oplus_{1 \leqslant i<j \leqslant s} \mathcal{O}\left(-D_{i}-D_{j}\right) \rightarrow \oplus_{1 \leqslant i \leqslant s} \mathcal{O}\left(-D_{i}\right) \rightarrow \mathcal{O} \rightarrow i_{*} \mathcal{O}_{Z} \rightarrow 0,
$$

we see that it suffices to prove that

$$
R \Gamma\left(\mathcal{O}_{Z}\left(D_{i_{1}}+\cdots+D_{i_{k}}\right)\right)=0
$$

for all $1 \leqslant i_{1}<\cdots<i_{k} \leqslant s$. Using that $\mathcal{O}_{Z}\left(D_{i}\right)$ has the form

$$
\mathcal{O} \otimes \cdots \otimes \mathcal{O} \otimes \mathcal{O}\left(-\psi_{\infty}\right) \otimes \mathcal{O}\left(-\psi_{0}\right) \otimes \mathcal{O} \cdots \otimes \mathcal{O}
$$

we conclude that this is indeed the case.

LEMma 4.9. The set $\hat{\mathbb{G}}$ is an exceptional collection with respect to the following order. Let $\mathcal{T}, \mathcal{T}^{\prime} \in \hat{\mathbb{G}}$. Let $\left(k_{1}, \ldots, k_{t} ; a_{1}, \ldots, a_{t}\right)$ and $\left(k_{1}^{\prime}, \ldots, k_{s}^{\prime} ; a_{1}^{\prime}, \ldots, a_{s}^{\prime}\right)$ be the corresponding data. Then $\mathcal{T}>\mathcal{T}^{\prime}$ if the sequence $\left(a_{1},-k_{1}, a_{2},-k_{2}, \ldots\right)$ is lexicographically (that is, alphabetically) larger than $\left(a_{1}^{\prime},-k_{1}^{\prime}, a_{2}^{\prime},-k_{2}^{\prime}, \ldots\right)$.

Proof. Let $Z$ and $Z^{\prime}$ be massive strata supporting sheaves $\mathcal{T}>\mathcal{T}^{\prime}$ in $\hat{\mathbb{G}}$. These sheaves have the form $R i_{Z *} \mathcal{L}$ and $R i_{Z^{\prime}{ }^{*}} \mathcal{L}^{\prime}$, respectively. We have to show that $R \operatorname{Hom}\left(\mathcal{T}, \mathcal{T}^{\prime}\right)=0$. Let $U$ be the smallest stratum containing both $Z$ and $Z^{\prime}$. Then $U$ is the intersection of boundary divisors $D_{1}, \ldots, D_{s}$ (these divisors are precisely the divisors containing both $Z$ and $Z^{\prime}$ ). We have

$$
R \operatorname{Hom}\left(R i_{Z *} \mathcal{L}, R i_{Z^{\prime}{ }_{*}} \mathcal{L}^{\prime}\right)=R \operatorname{Hom}\left(L j_{Z^{\prime}}{ }^{*} R j_{Z *} \mathcal{L}, \mathcal{L}^{\prime}\right) .
$$

By [Huy06, Corollary 11.4(i)], it suffices to prove that

$$
R \operatorname{Hom}\left(R j_{Z *} \mathcal{L}, R j_{Z^{\prime}}{ }_{*} \mathcal{L}^{\prime}(D)\right)=0
$$

for every $D=D_{i_{1}}+\cdots+D_{i_{k}}$ with $1 \leqslant i_{1}<\cdots<i_{k} \leqslant s$, where $j_{Z}$ and $j_{Z^{\prime}}$ denotes the embeddings of $Z$ and $Z^{\prime}$, respectively, into $U$. Let $W=Z \cap Z^{\prime}$. We can assume that $W$ is non-empty as otherwise there is nothing to prove. Let $i_{W, Z}: W \hookrightarrow Z$ and $i_{W, Z^{\prime}}: W \hookrightarrow Z^{\prime}$ be the inclusions. We note that $Z$ and $Z^{\prime}$ intersect transversally along $W$ in $U$, and therefore $j_{Z}$ and $j_{Z^{\prime}}$ are Tor-independent. Next we apply cohomology and base change:

$$
\begin{aligned}
R \operatorname{Hom}\left(R j_{Z *} \mathcal{L}, R j_{Z^{\prime}{ }_{*}} \mathcal{L}^{\prime}(D)\right) & =R \operatorname{Hom}\left(L j_{Z^{\prime}}{ }^{*} R j_{Z *} \mathcal{L}, \mathcal{L}^{\prime}(D)\right)=R \operatorname{Hom}\left(R i_{W, Z^{\prime}{ }_{*}} L i_{W, Z}^{*} \mathcal{L}, \mathcal{L}^{\prime}(D)\right) \\
& =R \operatorname{Hom}\left(L i_{W, Z}^{*} \mathcal{L}, L i_{W, Z^{\prime}} \mathcal{L}^{\prime}(D)\right),
\end{aligned}
$$

where for some morphism $f: X \rightarrow Y$, we denote the adjoint functor to $R f_{*}(-)$ by $L f^{!}(-)$. By Grothendieck duality, we have for $E \in D^{b}(Y)$ that $L f^{!}(E)=L f^{*}(E) \otimes \omega_{f}[\operatorname{dim}(f)]$. Here, $\omega_{f}=\omega_{X} \otimes f^{*} \omega_{Y}^{*}$ and $\operatorname{dim}(f)=\operatorname{dim}(X)-\operatorname{dim}(Y)$. So it suffices to prove that

$$
R \operatorname{Hom}\left(L i_{W, Z}^{*} \mathcal{L}, L i_{W, Z^{\prime}}^{*} \mathcal{L}^{\prime} \otimes\left(D+c_{1}(\mathcal{N})\right)\right)=0,
$$

where $c_{1}(\mathcal{N})$ is the first Chern class of the normal bundle $\mathcal{N}:=\mathcal{N}_{W, Z^{\prime}}$, that is, the sum of all boundary divisors that cut out $W$ inside $Z^{\prime}$ or, alternatively, the sum of boundary divisors that cut out $Z$ but do not contain $Z^{\prime}$. 


\section{DERIVED CATEGORY OF MODUli OF POINTED CURVES. I}

Now we proceed case by case. We write

$$
\begin{aligned}
& W=\overline{L M}_{K_{1}} \times \overline{L M}_{K_{2}} \times \cdots, \\
& R \operatorname{Hom}\left(L i_{W, Z}{ }^{*} \mathcal{L}, L i_{W, Z^{\prime}}{ }^{*} \mathcal{L}^{\prime}(D+N)\right)=C_{1} \otimes C_{2} \otimes \cdots,
\end{aligned}
$$

where $C_{1}$ is computed on $\overline{L M}_{K_{1}}$, etc. Note that if $N=N_{1} \sqcup \cdots \sqcup N_{t}$ and $N=N_{1}^{\prime} \sqcup \cdots \sqcup N_{t^{\prime}}^{\prime}$ are the two partitions corresponding to $\mathcal{T}$ and $\mathcal{T}^{\prime}$, respectively (hence, $\left|N_{i}\right|=k_{i}$ and $\left|N_{i}^{\prime}\right|=k_{i}^{\prime}$ for all $i$ ), then $W \neq \emptyset$ implies that either $N_{1} \subseteq N_{1}^{\prime}$ or $N_{1}^{\prime} \subseteq N_{1}$. In particular, we have $\left|K_{1}\right|=\min \left(k_{1}, k_{1}^{\prime}\right)$, and if $k_{1}=k_{1}^{\prime}$, then we have $N_{1}=N_{1}^{\prime}$.

Case 1. Suppose $a_{1}>a_{1}^{\prime}$. We would like to show that $C_{1}=0$.

If $k_{1}<k_{1}^{\prime}$, then $C_{1}=R \operatorname{Hom}\left(-G_{a_{1}},-G_{a_{1}^{\prime}}-\psi_{\infty}\right)$, where $-\psi_{\infty}$ is a contribution from $N$ (there is no contribution to $C_{1}$ from $\left.D\right)$. Hence, $C_{1}=0$ by Lemma $4.5(4)$.

If $k_{1}=k_{1}^{\prime}$, then there is no contribution from $c_{1}(\mathcal{N})$ to $C_{1}$, and we have that either $C_{1}=$ $R \operatorname{Hom}\left(-G_{a_{1}},-G_{a_{1}^{\prime}}\right)=0$ (if $D$ does not include $D_{K_{1}}$ ) or $C_{1}=R \operatorname{Hom}\left(-G_{a_{1}},-G_{a_{1}^{\prime}}-\psi_{\infty}\right)=0$ (if $D$ includes $D_{K_{1}}$ ).

Finally, if $k_{1}>k_{1}^{\prime}$, then there are no contributions from $c_{1}(\mathcal{N})$ or $D$ to $C_{1}$ and $C_{1}=$ $R \operatorname{Hom}\left(L,-G_{a_{1}^{\prime}}\right)=0$, where $L=-G_{a_{1}}$ if $a_{1}<k_{1}^{\prime}$ and $L=\mathcal{O}$ otherwise. In both cases, $C_{1}=0$ by Lemma 4.5 .

Case 2. Suppose $a_{1}=a_{1}^{\prime}$ and $k_{1}<k_{1}^{\prime}$. As in case 1 , we have that

$$
C_{1}=R \operatorname{Hom}\left(-G_{a_{1}},-G_{a_{1}}-\psi_{\infty}\right)=0 \text {. }
$$

Case 3. Suppose $a_{1}=a_{1}^{\prime}, k_{1}=k_{1}^{\prime}$ and that $D$ includes $D_{K_{1}}$.

In this case also, $C_{1}=R \operatorname{Hom}\left(-G_{a_{1}},-G_{a_{1}}-\psi_{\infty}\right)=0$.

Case 4. Suppose $a_{1}=a_{1}^{\prime}, k_{1}=k_{1}^{\prime}$ and that $D$ does not include $D_{K_{1}}$.

In this case, $C_{1}=R \operatorname{Hom}\left(-G_{a_{1}},-G_{a_{1}}\right)=\mathbb{C}$ is useless. However, we can now proceed exactly as above, restricting to the next Losev-Manin factor $\overline{L M}_{K_{2}}$ in $W$. Note that, in general, the factors $\overline{L M}_{K_{i}}$ appearing in $W$ need not be positive-dimensional, but in this case, since $K_{1}=K_{1}^{\prime}$, we must have that $\left|K_{2}\right| \geqslant 2$, and we can proceed by induction. The lemma follows.

The Cremona action gives another possible linear order.

COROLlary 4.10. The set $\hat{\mathbb{G}}$ is an exceptional collection with respect to the order $<^{\prime}$ :

$$
\left(k_{1}, \ldots, k_{t} ; a_{1}, \ldots, a_{t}\right)>^{\prime}\left(k_{1}^{\prime}, \ldots, k_{t}^{\prime} ; a_{1}^{\prime}, \ldots, a_{t}^{\prime}\right)
$$

if the sequence $\left(k_{t}-a_{t},-k_{t}, k_{t-1}-a_{t-1},-k_{t-1}, \ldots\right)$ is lexicographically larger than $\left(k_{s}^{\prime}-a_{s}^{\prime},-k_{s}^{\prime}\right.$, $\left.k_{s-1}^{\prime}-a_{s-1}^{\prime},-k_{s-1}^{\prime}, \ldots\right)$.

Remark 4.11. The linear orders $<$ and $<^{\prime}$ are clearly not $\left(S_{2} \times S_{N}\right)$-equivariant. The lemma shows that both orders refine the $\left(S_{2} \times S_{N}\right)$-equivariant relation $\prec$ given by paths in the quiver with arrows

$$
\mathcal{T} \rightarrow \mathcal{T}^{\prime} \Longleftrightarrow R \operatorname{Hom}\left(\mathcal{T}, \mathcal{T}^{\prime}\right) \neq 0 .
$$

In other words, this quiver has no cycles. It would be nice to describe it combinatorially. It would 


\section{A.-M. Castravet and J. Tevelev}

be even better to explicitly describe the algebra

$$
\bigoplus_{\mathcal{T} \prec \mathcal{T}^{\prime}} R \operatorname{Hom}\left(\mathcal{T}, \mathcal{T}^{\prime}\right)
$$

Here are some easy observations about the quiver:

(1) If there is an arrow between $\mathcal{T}$ and $\mathcal{T}^{\prime}$, then the corresponding strata have a non-empty intersection.

(2) The line bundles can be arranged to be at the right of torsion sheaves in the collection: for any torsion sheaf $\mathcal{T}^{\prime}$ in $\hat{\mathbb{G}}$ and any line bundle $\mathcal{T}=G_{a}^{\vee}$, we have (in the notation of the proof of Lemma 4.9)

$$
R \operatorname{Hom}\left(\mathcal{T}, \mathcal{T}^{\prime}\right)=R \Gamma\left(G_{a \mid Z^{\prime}} \otimes \mathcal{T}^{\prime}\right)=C_{1} \otimes C_{2} \otimes \cdots
$$

and $C_{1}=R \operatorname{Hom}\left(L, G_{a_{1}^{\prime}}^{\vee}\right)$, where $L=G_{a_{1}}$ if $a_{1}<k_{1}^{\prime}$ and $L=\mathcal{O}$ otherwise. In both cases, $C_{1}=0$ by Lemma 4.5 .

(3) It is not true in general that sheaves can be pre-ordered by codimension of support. For example, on $\overline{L M}_{8}$, the sheaf $\mathcal{T}^{\prime}$ with data $(3,2,3 ; 2,1,1)$ and support $Z^{\prime}$ has to be to the right of the sheaf $\mathcal{T}$ with data $(3,5 ; 1,3)$ and support $Z$ with $Z^{\prime} \subseteq Z$, as an easy computation as above shows that $R \operatorname{Hom}\left(\mathcal{T}, \mathcal{T}^{\prime}\right) \neq 0$.

Let us give more information about the quiver. We first introduce some terminology.

DeFinition 4.12. Let $\mathcal{T} \in \hat{\mathbb{G}}$ with support $Z$ :

$$
\begin{gathered}
Z=\overline{L M}_{K_{1}} \times \overline{L M}_{K_{2}} \times \cdots \times \overline{L M}_{K_{t}}, \\
\mathcal{T}=G_{a_{1}}^{\vee} \cdots \otimes G_{a_{t}}^{\vee} .
\end{gathered}
$$

(1) We call $\overline{L M}_{K_{1}}$ the first component of $Z, \overline{L M}_{K_{2}}$ the second component of $Z$, etc. and $\overline{L M}_{K_{t}}$ the last component of $Z$.

(2) We say that we remove the component $\overline{L M}_{K_{i}}$ from $\mathcal{T}$ if we consider the sheaf $\tilde{\mathcal{T}}$ given by

$$
\begin{gathered}
\tilde{Z}=\overline{L M}_{K_{1}} \times \cdots \times \overline{L M}_{K_{i-1}} \times \overline{L M}_{K_{i+1}} \times \cdots \times \overline{L M}_{K_{t}}, \\
\tilde{\mathcal{T}}=G_{a_{1}}^{\vee} \cdots \otimes G_{a_{i-1}}^{\vee} \otimes G_{a_{i+1}}^{\vee} \otimes \cdots G_{a_{t}}^{\vee} .
\end{gathered}
$$

(3) We say that the end data of $\mathcal{T}$ is $\left(k_{1}, k_{t} ; k_{1}-a_{1}, a_{t}\right)$. Clearly, different objects in $\hat{\mathbb{G}}$ could have the same end data.

(4) Recall from the proof of Lemma 4.9 that to show that $R \operatorname{Hom}\left(\mathcal{T}, \mathcal{T}^{\prime}\right)=0$, it suffices to show that

$$
R \operatorname{Hom}\left(L i_{W, Z}^{*} \mathcal{T}, L i_{W, Z^{\prime}}^{*} \mathcal{T}^{\prime} \otimes(N+D)\right)=0,
$$

where $W=Z \cap Z^{\prime}, N$ is the first Chern class of the normal bundle $\mathcal{N}_{W, Z^{\prime}}$, that is, the sum of boundary divisors that cut out $Z$, and $D=D_{i_{1}}+\cdots+D_{i_{r}}$ is a (possibly empty) sum of boundary divisors containing both $Z$ and $Z^{\prime}$. We let

$$
\begin{gathered}
W=\overline{L M}_{S_{1}} \times \overline{L M}_{S_{2}} \times \cdots, \\
R \operatorname{Hom}\left(i_{W, Z}{ }^{*} \mathcal{T}, i_{W, Z^{\prime}}{ }^{*} \mathcal{T}^{\prime}(N+D)\right)=C_{S_{1}} \otimes C_{S_{2}} \otimes \cdots .
\end{gathered}
$$

In what follows, we will refer to the $C_{S_{i}}$ as the components of $R \operatorname{Hom}\left(\mathcal{T}, \mathcal{T}^{\prime}\right)$.

Lemmas 4.9 and 4.10 have the following corollary, which can be used as an algorithm to determine, given a pair of torsion objects $\mathcal{T}$ and $\mathcal{T}^{\prime}$ in $\hat{\mathbb{G}}$, whether $R \operatorname{Hom}\left(\mathcal{T}, \mathcal{T}^{\prime}\right)=0$ or $R \operatorname{Hom}\left(\mathcal{T}^{\prime}, \mathcal{T}\right)=0$ 


\section{DERIVED CATEGORY OF MODUli OF POINTED CURVES. I}

Corollary 4.13. Let $\mathcal{T}$ and $\mathcal{T}^{\prime}$ be torsion sheaves in $\hat{\mathbb{G}}$ with supports $Z$ and $Z^{\prime}$ and end data $\left(k_{1}, k_{t} ; b_{1}, b_{t}\right)$ and $\left(k_{1}^{\prime}, k_{s}^{\prime} ; b_{1}^{\prime}, b_{s}^{\prime}\right)$. If the inequalities

$$
b_{1}+b_{t} \leqslant b_{1}^{\prime}+b_{s}^{\prime}, \quad k_{1}+k_{t}-b_{1}-b_{t} \geqslant k_{1}^{\prime}+k_{s}^{\prime}-b_{1}^{\prime}-b_{s}^{\prime}
$$

both hold and one of them is a strict inequality, then $R \operatorname{Hom}\left(\mathcal{T}, \mathcal{T}^{\prime}\right)=0$. Moreover, if both inequalities are equalities, then $R \operatorname{Hom}\left(\mathcal{T}, \mathcal{T}^{\prime}\right) \neq 0$ is only possible when

$$
b_{1}=b_{1}^{\prime}, \quad b_{t}=b_{s}^{\prime}, \quad k_{1}=k_{1}^{\prime}, \quad k_{t}=k_{s}^{\prime}
$$

and the first and last components are the same, that is, $K_{1}=K_{1}^{\prime}$ and $K_{t}=K_{s}^{\prime}$. Whenever all these conditions hold, we have that

$$
R \operatorname{Hom}\left(\mathcal{T}, \mathcal{T}^{\prime}\right)=0 \quad \text { if } \quad R \operatorname{Hom}\left(\tilde{\mathcal{T}}, \tilde{\mathcal{T}}^{\prime}\right)=0,
$$

where $\tilde{\mathcal{T}}$ and $\tilde{\mathcal{T}}^{\prime}$ are the sheaves obtained from $\mathcal{T}$ and $\mathcal{T}^{\prime}$, respectively, after removing the first and last components $\overline{L M}_{K_{1}}$ and $\overline{L M}_{K_{t}}$.

Proof. Recall that we have

$$
a_{1}=k_{1}-b_{1}, \quad a_{1}^{\prime}=k_{1}^{\prime}-b_{1}^{\prime}, \quad a_{t}=b_{t}, \quad a_{s}^{\prime}=b_{s}^{\prime} .
$$

By Lemma 4.9 , if $k_{1}-b_{1}>k_{1}^{\prime}-b_{1}^{\prime}$, then $R \operatorname{Hom}\left(\mathcal{T}, \mathcal{T}^{\prime}\right)=0$. Similarly, by Lemma 4.10, if $k_{t}-b_{t}>k_{s}^{\prime}-b_{s}^{\prime}$, then $R \operatorname{Hom}\left(\mathcal{T}, \mathcal{T}^{\prime}\right)=0$. Since we assume

$$
\left(k_{1}-b_{1}\right)+\left(k_{t}-b_{t}\right) \geqslant\left(k_{1}^{\prime}-b_{1}^{\prime}\right)+\left(k_{s}^{\prime}-b_{s}^{\prime}\right),
$$

it follows that we must have $k_{1}-b_{1}=k_{1}^{\prime}-b_{1}^{\prime}, k_{t}-b_{t}=k_{s}^{\prime}-b_{s}^{\prime}$. Now if $-k_{1}>-k_{1}^{\prime}$, again by Lemma 4.9 , we have $R \operatorname{Hom}\left(\mathcal{T}, \mathcal{T}^{\prime}\right)=0$. Similarly, if $-k_{t}>-k_{s}^{\prime}$, by Lemma 4.10 , we have $R \operatorname{Hom}\left(\mathcal{T}, \mathcal{T}^{\prime}\right)=0$. Hence, we may assume $k_{1} \geqslant k_{1}^{\prime}$ and $k_{t} \geqslant k_{s}^{\prime}$. But then $\left(k_{1}+k_{1}^{\prime}\right)-\left(k_{t}+k_{s}^{\prime}\right) \geqslant 0$, while

$$
\left(k_{1}+k_{1}^{\prime}\right)-\left(k_{t}+k_{s}^{\prime}\right)=\left(b_{1}+b_{t}\right)-\left(b_{1}^{\prime}+b_{s}^{\prime}\right) \geqslant 0 .
$$

Hence, we must have $k_{1}=k_{1}^{\prime}$ and $k_{t}=k_{s}^{\prime}$ and hence $b_{1}=b_{1}^{\prime}$ and $b_{t}=b_{s}^{\prime}$.

If these equalities hold, for the intersection $Z \cap Z^{\prime}$ to be non-empty, we must have that the first and last components are the same, that is, $K_{1}=K_{1}^{\prime}$ and $K_{t}=K_{s}^{\prime}$. As in the proof of Lemma 4.9 (case 4), we can remove the first and last components, $\overline{L M}_{K_{1}}$ and $\overline{L M}_{K_{t}}$, from $Z$ and $Z^{\prime}$ and proceed with the rest.

We finish this section by relating line bundles $G_{1}, \ldots, G_{n-1}$ on $\overline{L M}_{n}$ to the wonderful compactification of $\mathrm{PGL}_{n}$. Following [Bri07], we identify Pic $\overline{\mathrm{PGL}_{n}}$ with the weight lattice of $\mathrm{PGL}_{n}$. Let $\alpha_{1}, \ldots, \alpha_{n-1}$ be simple roots, and let $\omega_{1}, \ldots, \omega_{n-1}$ be fundamental co-weights. It is shown in [Bri07] that $\alpha_{1}, \ldots, \alpha_{n-1}$ and $\omega_{1}, \ldots, \omega_{n-1}$ span the effective cone and the nef cone, respectively, of $\overline{\mathrm{PGL}}_{n}$. We identify $\overline{L M}_{n}$ with the closure of the maximal torus in $\mathrm{PGL}_{n}$.

Proposition 4.14. Divisors on $\overline{\mathrm{PGL}_{n}}$ corresponding to $\omega_{1}, \ldots, \omega_{n-1}$ restrict to divisors $G_{1}, \ldots, G_{n-1}$ on the Losev-Manin space $\overline{L M}_{n}$.

Proof. First we consider divisors $D_{1}, \ldots, D_{n}$ on $\overline{\mathrm{PGL}_{n}}$ which correspond to simple roots $\alpha_{1}, \ldots, \alpha_{n-1}$. We will show that they restrict to total boundary divisors

$$
\Delta_{1}=\sum \delta_{0 i}, \quad \ldots, \quad \Delta_{n-1}=\sum \delta_{0 i_{1} \ldots i_{n-1}}
$$

on the Losev-Manin space $\overline{L M}_{n}$. Indeed, it is known (see, for example, [Bri07]) that $D_{1}, \ldots, D_{n-1}$ are the boundary divisors of $\overline{\mathrm{PGL}_{n}}$, that is,

$$
\overline{\mathrm{PGL}_{n}} \backslash \mathrm{PGL}_{n}=D_{1} \cup \cdots \cup D_{n-1} .
$$




\section{A.-M. Castravet and J. Tevelev}

In particular, every $D_{i}$ restricts to a linear combination of boundary divisors of $\overline{L M}_{n}$. Since each of these divisors is $\mathrm{PGL}_{n}$-invariant (acting by conjugation), the restriction is invariant under $S_{n}$ (= Weyl group); that is, it is a linear combination of total boundary divisors.

The compactification $\overline{\mathrm{PGL}_{n}}$ is a spherical homogeneous space for the group $\mathrm{PGL}_{n} \times \mathrm{PGL}_{n}$, extending its action on $\mathrm{PGL}_{n}$ by left and right translations. The group of semi-invariant functions $\Lambda=k\left(\mathrm{PGL}_{n}\right)^{(\mathcal{B})} / k^{*}$ (where $\mathcal{B}=B^{-} \times B$ is a Borel subgroup of $\mathrm{PGL}_{n} \times \mathrm{PGL}_{n}$ ) is identified with the root lattice of $\mathrm{PGL}_{n}$, which in turn is identified with the lattice of characters $M=$ $k(T)^{(T)} / k^{*}$ of the maximal torus in $\mathrm{PGL}_{n}$ via the restriction of the $\mathcal{B}$-semi-invariant functions. Every boundary divisor $D_{i}$ determines a functional $\rho\left(D_{i}\right): \Lambda \rightarrow \mathbb{Z}$ (and so an element of the dual weight lattice $\Lambda^{*}=\mathbb{Z}^{n} /\langle 1, \ldots, 1\rangle$ ) by taking a divisorial valuation of a function in $\Lambda$ along $D_{i}$. In fact, we have $\rho\left(D_{i}\right)=\omega_{i}=e_{1}+\cdots+e_{i} \bmod \langle 1, \ldots, 1\rangle$, the fundamental coweight (see, for example, [Bri07]). These vectors span the Weyl chamber, and the fan of $\overline{L M}_{n}$ (as a toric variety) is precisely the fan of its Weyl group translates. Moreover, vectors $\omega_{i}$ are primitive vectors along the rays which give boundary divisors $\delta_{0,1, \ldots, i}$; see [LM00]. So we are done by [BK05, Lemma 6.1.6].

By pulling back a coordinate hyperplane in $\mathbb{P}^{n-1}$ and symmetrizing, we get the formula

$$
\psi_{0}=\frac{n-1}{n} \Delta_{1}+\cdots+\frac{1}{n} \Delta_{n-1} .
$$

Combining it with Definition 1.9 yields the following formula for the $G_{i}$ :

$$
G_{i}=\sum_{j=1}^{n-1} B_{i j} \Delta_{j},
$$

where $B_{i j}=i(n-j) / n$ if $i \leqslant j$ and $B_{i j}=B_{j i}$ if $i>j$. It is well known and easy to prove that the inverse of the matrix $B$ is the Cartan matrix of the root system $A_{n-1}$, and therefore $\psi_{i}=\sum_{j=1}^{n-1} B_{i j} \alpha_{j}$, which finishes the proof.

\section{Fullness of the exceptional collection $\widehat{\mathbb{G}}$}

We will need the following more general set-up.

Definition 5.1. For every integer $r \geqslant-1$, define a contravariant functor $X^{r}$ from $\mathbb{N}$ to the category of smooth projective varieties as follows. Let $X_{N}^{r}$ be an iterated blow-up of $\mathbb{P}^{n+r}$ in $n$ general points (marked by $N$ ) followed by the blow-up of the $\left(\begin{array}{l}n \\ 2\end{array}\right)$ proper transforms of the lines passing through two points, the $\left(\begin{array}{l}n \\ 3\end{array}\right)$ proper transforms of the planes passing through three points, etc. For example,

$$
X_{N}^{-1}=\overline{L M}_{N}, \quad X_{\emptyset}^{r}=\mathbb{P}^{r} .
$$

For every $M \subseteq N$, the forgetful morphism $\pi_{N \backslash M}: X_{N}^{r} \rightarrow X_{M}^{r}$ is induced by a linear projection from points in $N \backslash M$.

For every subset $S \subseteq N$ of cardinality at most $n+r-1$, we denote by $E_{S} \subseteq X_{N}^{r}$ the exceptional divisor over a subspace spanned by points in $S$.

Proposition 5.2. All conditions of Theorem 3.5 are satisfied. Thus we have a semi-orthogonal decomposition

$$
D^{b}\left(X_{S}^{r}\right)=\left\langle D_{\text {cusp }}^{b}\left(X_{S}^{r}\right),\left\{L \pi_{K}^{*} D_{\text {cusp }}^{b}\left(X_{S \backslash K}^{r}\right)\right\}_{K \subset S}, L \pi_{N}^{*} D^{b}\left(\mathbb{P}^{r}\right)\right\rangle,
$$

where $K$ runs over proper subsets of $S$ in order of increasing cardinality. 


\section{DERIVED CATEGORY OF MODULI OF POINTED CURVES. I}

Notation 5.3. For every $i \in N$, we have a birational morphism

$$
f_{i}: X_{N}^{r} \rightarrow X_{N \backslash i}^{r+1}
$$

obtained by blowing down exceptional divisors $E_{S}$, for $i \in S$, in the order of decreasing cardinality.

Definition 5.4 (Strata in $\boldsymbol{X}_{\boldsymbol{N}}^{\boldsymbol{r}}$ ). Consider partitions

$$
N=N_{1} \sqcup \cdots \sqcup N_{k}, \quad\left|N_{u}\right|>0 \quad(u=1, \ldots, k-1) .
$$

Set

$$
Z_{N_{1}, \ldots, N_{k}}=E_{N_{1}} \cap E_{N_{1} \cup N_{2}} \cap \cdots \cap E_{N_{1} \cup \cdots \cup N_{k-1}} .
$$

We call $Z_{N_{1}, \ldots, N_{k}}$ a stratum in $X_{N}^{r}$. We call a stratum in $X_{N}^{r}$ massive if it is the image of a massive stratum in $\overline{L M}_{n+r+1}$ via the birational map $\overline{L M}_{n+r+1} \rightarrow X_{N}^{r}$ which is the composition of the maps $f_{i}$ for those $i \notin N$.

For $r \geqslant 0$, each stratum $Z_{N_{1}, \ldots, N_{k}}$ is the image of a stratum in $\overline{L M}_{n+r+1}$. For all $r \geqslant-1$, we have an identification

$$
Z_{N_{1}, \ldots, N_{k}} \simeq \overline{L M}_{N_{1}} \times \cdots \times \overline{L M}_{N_{k-1}} \times X_{N_{k}}^{r}
$$

where $X_{N_{k}}^{r}$ is the blow-up of $\mathbb{P}^{\left|N_{k}\right|+r}$ at the linear subspaces spanned by the points in $N_{k}$. If $r \geqslant 0$, a stratum $Z_{N_{1}, \ldots, N_{k}}$ is massive if and only if $\left|N_{u}\right| \geqslant 2$ for all $u=1, \ldots, k-1$ and $\left|N_{k}\right|+r>0$.

Definition 5.5. We let $\hat{\mathbb{G}}_{N}^{r}$ be a collection of objects in $D^{b}\left(X_{N}^{r}\right)$ defined inductively as follows:

$$
\hat{\mathbb{G}}_{N}^{-1}:=\hat{\mathbb{G}}_{N}, \quad \hat{\mathbb{G}}_{N}^{r+1}=R f_{i_{*}}\left(\hat{\mathbb{G}}_{N}^{r}\right) .
$$

Definition 5.6. Consider the following line bundles on $X_{N}^{r}($ for $r \geqslant-1)$ :

$$
G_{a}^{r \vee}=-a H+(a-1) \sum_{i \in N} E_{i}+(a-2) \sum_{i, j \in N} E_{i j}+\cdots \quad(a=1, \ldots, n+r)
$$

(the coefficient in front of the exceptional divisor must be positive).

Lemma 5.7. For every $E \in D_{\text {cusp }}^{b}\left(X_{N}^{r}\right)$, we have $R f_{i_{*}}(E) \in D_{\text {cusp }}^{b}\left(X_{N \backslash i}^{r+1}\right)$. In particular, the collection $\hat{\mathbb{G}}_{N}^{r}$ is contained in $D_{\text {cusp }}^{b}\left(X_{N}^{r}\right)$. Moreover, we have

$$
R f_{i_{*}} G_{a}^{r \vee}=G_{a}^{r+1 \vee} \quad(a=1, \ldots, n+r) .
$$

In particular, $\hat{\mathbb{G}}_{N}^{r}$ contains the line bundles $G_{a}^{r \vee}($ for $1 \leqslant a \leqslant n+r)$ and the following torsion objects:

$$
\mathcal{T}=\left(i_{Z}\right)_{*} \mathcal{L}, \quad \mathcal{L}=G_{a_{1}}^{\vee} \otimes \cdots \otimes G_{a_{k-1}}^{\vee} \otimes G_{a_{k}}^{r \vee}
$$

for all massive strata $Z=Z_{N_{1}, \ldots, N_{k}}$ in $X_{N}^{r}$, all $1 \leqslant a_{u} \leqslant\left|N_{u}\right|-1$ for $u=1, \ldots, k-1$ and all $1 \leqslant a_{k} \leqslant\left|N_{k}\right|+r$.

Proof. The first statement follows from the commutative diagram

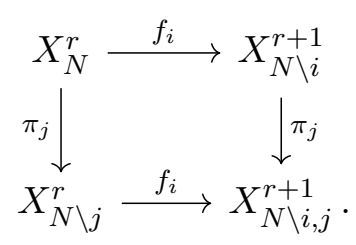




\section{A.-M. Castravet and J. Tevelev}

To prove the rest of the lemma, it suffices to prove $R f_{i_{*}} G_{a}^{r \vee}=G_{a}^{r+1}{ }^{\vee}$. Set $t=\min \{a-1, n\}$. Note that

$$
\begin{aligned}
L f_{i}^{*} G_{a}^{r+1 \vee}=f_{i}^{*} G_{a}^{r+1 \vee}= & -a H+(a-1) \sum_{j \in N \backslash\{i\}} E_{j} \\
& +(a-2) \sum_{j, k \in N \backslash\{i\}} E_{j k}+\cdots+(a-t) \sum_{J \subseteq N \backslash\{i\},|J|=t} E_{J},
\end{aligned}
$$

as the pull-backs $f_{i}^{*} E_{J}$ are simply the proper transforms of the divisors $E_{J}$ under the blow-up map $f_{i}$. In particular, $f_{i}^{*} G_{a}^{r+1 \vee}=G_{a}^{r+1 \vee}+F$, where

$$
F=(a-1) E_{i}+(a-2) \sum_{j \in N \backslash\{i\}} E_{i j}+\cdots+(a-t) \sum_{J \subseteq N, i \in J,|J|=t} E_{J} .
$$

Note that the coefficient $a-|J|$ of any $E_{J}$ appearing in $F$ satisfies

$$
1 \leqslant a-|J| \leqslant n+r-j<n+r-j+1=\operatorname{codim}_{X_{N}^{r}} E_{J} .
$$

This implies, after repeatedly applying Lemma 5.8 to the morphisms that successively blow down the divisors $E_{J}$ with $i \in J$, for a fixed $|J|$ (starting from the larger $|J|$ to the smaller), that $R f_{i *} \mathcal{O}(F)=\mathcal{O}$. It follows that

$$
R f_{i_{*}} G_{a}^{r \vee}=R f_{i_{*}}\left(f_{i}^{*} G_{a}^{r+1 \vee} \otimes \mathcal{O}(F)\right)=G_{a}^{r+1 \vee} \otimes R f_{i_{*}} \mathcal{O}(F)=G_{a}^{r+1}{ }^{2} .
$$

The following lemma is well known.

Lemma 5.8. Let $p: X \rightarrow Y$ be a blow-up of a smooth subvariety $Z$ of codimension $r+1$ of a smooth projective variety $Y$. Let $E$ be the exceptional divisor. Then for all $1 \leqslant i \leqslant r$, we have

$$
R p_{*} \mathcal{O}_{X}(i E)=\mathcal{O}_{Y}
$$

Lemma 5.9. All sheaves in $\hat{\mathbb{G}}_{N}^{r}$ are exceptional.

The proof is identical to that of Lemma 4.8, and we omit it. The same direct computation as in Lemma 4.3 shows that the line bundles $G_{a}^{r}$ satisfy the same restriction properties as the line bundles $G_{a}$.

Lemma 5.10. For $S \subseteq N$, identifying the exceptional divisor $E_{S} \subseteq X_{N}^{r}$ with the product $\overline{L M}_{S} \times X_{N \backslash S}^{r}$, we have

$$
G_{a \mid E_{S}}^{r}= \begin{cases}G_{a} \otimes \mathcal{O} & \text { if } a<|S|, \\ \mathcal{O} & \text { if } a=|S|, \\ \mathcal{O} \otimes G_{a-|S|}^{r} & \text { if } a>|S| .\end{cases}
$$

The analogue of Lemma 4.5 is the following.

Lemma 5.11. (1) Every $G_{a}^{r}$ is nef (hence, globally generated), of relative degree 1 with respect to any forgetful map $\pi_{i}$, for $i \in N$.

(2) Each $\left(G_{a}^{r}\right)^{\vee}$ is acyclic.

(3) We have $R \operatorname{Hom}\left(G_{a}, G_{b}\right)=0$ if $a>b$.

(4) We have $R \Gamma\left(-\psi_{0}+G_{a}-G_{b}\right)=0$ if $a<b$.

The proof is identical to that of Lemma 4.5, and we omit it. Note that unlike in the case $r=$ -1 , when $r \geqslant 0$, there is no more $S_{2}$-symmetry, and it is generally false that $R \operatorname{Hom}\left(G_{a}, G_{b}\right)=0$ 


\section{DERIVED CATEGORY OF MODUli OF POINTED CURVES. I}

if $a<b$. For example, if $r \geqslant 0$, then $R \operatorname{Hom}\left(G_{1}, G_{2}\right)=R \Gamma\left(H-\sum_{i \in N} E_{i}\right) \neq 0$. As a result, the order < of Lemma 4.9 does not generally descend to an order on $\hat{\mathbb{G}}_{N}^{r}$ which makes $\hat{\mathbb{G}}_{N}^{r}$ an exceptional collection (for example, if $N=\emptyset$ ). However, the order $<^{\prime}$ from Corollary 4.10 descends to an order on $\hat{\mathbb{G}}_{N}^{r}$ which makes $\hat{\mathbb{G}}_{N}^{r}$ an exceptional collection.

Lemma 5.12. For all $r \geqslant-1$, the set $\hat{\mathbb{G}}_{N}^{r}$ is an exceptional collection with respect to the following order. Let $\mathcal{T}, \mathcal{T}^{\prime} \in \hat{\mathbb{G}}_{N}^{r}$. Let $\left(k_{1}, \ldots, k_{t} ; a_{1}, \ldots, a_{t}\right)$ and $\left(k_{1}^{\prime}, \ldots, k_{s}^{\prime} ; a_{1}^{\prime}, \ldots, a_{s}^{\prime}\right)$ be the corresponding data. Then $\mathcal{T}>^{\prime} \mathcal{T}^{\prime}$ if the sequence

$$
\left(k_{t}-a_{t},-k_{t}, k_{t-1}-a_{t-1},-k_{t-1}, \ldots\right)
$$

is lexicographically (that is, alphabetically) larger than

$$
\left(k_{s}^{\prime}-a_{s}^{\prime},-k_{s}^{\prime}, k_{s-1}^{\prime}-a_{s-1}^{\prime},-k_{s-1}^{\prime}, \ldots\right) .
$$

Proof. The proof is similar to that of Lemma 4.9. We sketch the proof for completeness. Let $Z$ and $Z^{\prime}$ be massive strata supporting sheaves $\mathcal{T}>\mathcal{T}^{\prime}$ in $\hat{\mathbb{G}}$. These sheaves have the form $R i_{Z *} \mathcal{L}$ and $R i_{Z^{\prime}{ }_{*}} \mathcal{L}^{\prime}$, respectively. We have to show that $R \operatorname{Hom}\left(\mathcal{T}, \mathcal{T}^{\prime}\right)=0$. Let $U$ be the smallest stratum containing both $Z$ and $Z^{\prime}$. Then $U$ is the intersection of codimension 1 strata (exceptional divisors) $D_{1}, \ldots, D_{s}$ containing both $Z$ and $Z^{\prime}$. Let $W=Z \cap Z^{\prime}$. We can assume that $W$ is nonempty as otherwise there is nothing to prove. Let $i_{W, Z}: W \hookrightarrow Z$ and $i_{W, Z^{\prime}}: W \hookrightarrow Z^{\prime}$ be the inclusions. As in the proof of Lemma 4.9, it suffices to prove that

$$
R \operatorname{Hom}\left(L i_{W, Z}^{*} \mathcal{L}, L i_{W, Z^{\prime}}^{*} \mathcal{L}^{\prime}\left(D+c_{1}(\mathcal{N})\right)\right)=0,
$$

where $c_{1}(\mathcal{N})$ is the first Chern class of the normal bundle $\mathcal{N}:=\mathcal{N}_{W, Z^{\prime}}$, that is, the sum of all the exceptional that cut out $Z$ but do not contain $Z^{\prime}$. We write

$$
\begin{gathered}
W=\overline{L M}_{K_{1}} \times \overline{L M}_{K_{2}} \times \cdots \times \overline{L M}_{K_{s-1}} \times X_{K_{t}}^{r}, \\
R \operatorname{Hom}\left(L i_{W, Z}{ }^{*} \mathcal{L}, L i_{W, Z^{\prime}}{ }^{*} \mathcal{L}^{\prime}\left(D+c_{1}(\mathcal{N})\right)\right)=C_{1} \otimes C_{2} \otimes \cdots \otimes C_{t},
\end{gathered}
$$

where $C_{1}$ is $R$ Hom between components of line bundles $L i_{W, Z}{ }^{*} \mathcal{L}$ and $L i_{W, Z^{\prime}}{ }^{*} \mathcal{L}^{\prime}\left(D+c_{1}(\mathcal{N})\right)$ corresponding to $\overline{L M}_{K_{1}}$, etc.

Case 1. Suppose $k_{t}-a_{t}>k_{t}^{\prime}-a_{t}^{\prime}$. We prove that $C_{t}=0$. If $k_{t}<k_{t}^{\prime}$, then $a_{t}^{\prime}>a_{t}+\left(k_{t}^{\prime}-k_{t}\right)>$ $\left(k_{t}^{\prime}-k_{t}\right)$. Hence,

$$
C_{t}=R \operatorname{Hom}\left(-G_{a_{t}},-G_{a_{t}^{\prime}-\left(k_{t}^{\prime}-k_{t}\right)}-\psi_{\infty}\right),
$$

where $-\psi_{0}$ is a contribution from $c_{1}(\mathcal{N})$ (there is no contribution from $D$ ). As $a_{t}^{\prime}-\left(k_{t}^{\prime}-k_{t}\right)>a_{t}$, it follows that $C_{t}=0$ by Lemma $5.11(4)$.

If $k_{t}=k_{t}^{\prime}$, then $a_{t}^{\prime}>a_{t}$. As there is no contribution from $c_{1}(\mathcal{N})$ to $C_{t}$, we have that either $C_{t}=R \operatorname{Hom}\left(-G_{a_{t}},-G_{a_{t}^{\prime}}\right)=0$ (if $D$ does not include $D_{K_{1}}$ ), or $C_{t}=R \operatorname{Hom}\left(-G_{a_{t}},-G_{a_{t}^{\prime}}-\psi_{0}\right)=0$ (if $D$ includes $D_{K_{1}}$ ).

If $k_{t}>k_{t}^{\prime}$, then there are no contributions from $c_{1}(\mathcal{N})$ or $D$ to $C_{t}$ and

$$
C_{t}=R \operatorname{Hom}\left(L,-G_{a_{t}^{\prime}}\right)=0,
$$

where $L=-G_{a_{t}-\left(k_{t}-k_{t}^{\prime}\right)}$ if $a_{t}>k_{t}-k_{t}^{\prime}$ and $L=\mathcal{O}$ otherwise. As, by assumption, $a_{t}-\left(k_{t}-k_{t}^{\prime}\right)<a_{t}^{\prime}$, it follows that in both cases, $C_{t}=0$ by Lemma 4.5 .

Case 2. Suppose $k_{t}-a_{t}=k_{t}^{\prime}-a_{t}^{\prime}$ and $k_{t}<k_{t}^{\prime}$. Then $a_{t}^{\prime}=\left(k_{t}^{\prime}-k_{t}\right)+a_{t}$ and hence $a_{t}^{\prime}>\left(k_{t}^{\prime}-k_{t}\right)$. As in case 1 , we have

$$
C_{t}=R \operatorname{Hom}\left(-G_{a_{t}},-G_{a_{t}^{\prime}-\left(k_{t}^{\prime}-k_{t}\right)}-\psi_{0}\right)=R \operatorname{Hom}\left(-G_{a_{t}},-G_{a_{t}}-\psi_{0}\right)=0 .
$$




\section{A.-M. Castravet and J. Tevelev}

Case 3. Suppose $a_{t}=a_{t}^{\prime}, k_{t}=k_{t}^{\prime}$ and that $D$ includes $D_{K_{t}}$. In this case, we have

$$
C_{t}=R \operatorname{Hom}\left(-G_{a_{t}},-G_{a_{t}}-\psi_{0}\right)=0 \text {. }
$$

Case 4. Suppose $a_{t}=a_{t}^{\prime}, k_{t}=k_{t}^{\prime}$ and that $D$ does not include $D_{K_{t}}$. In this case,

$$
C_{t}=R \operatorname{Hom}\left(-G_{a_{t}},-G_{a_{t}}\right)=\mathbb{C}
$$

is useless. However, we can now use Corollary 4.10 as the remaining factors are Losev-Manin spaces (or, alternatively, proceed exactly as above, by restricting to the next Losev-Manin factor $\overline{L M}_{K_{t-1}}$ in $W$ ). The lemma follows.

Lemma 5.13. Let $r \geqslant-1$. For every $\mathcal{T} \in \hat{\mathbb{G}}_{N}^{r+1}$ and every $j \in N$, we have

$$
R \pi_{j_{*}} L f_{i}{ }^{*} \mathcal{T}=0 .
$$

Proof. We use the commutative diagram (5.1). Since $\pi_{j}$ is flat and $X_{N \backslash j}^{r}{ }^{\times} X_{N \backslash i j}^{r+1} X_{N \backslash i}^{r+1}$ has toroidal, and hence rational, singularities, the claim follows by cohomology and base change.

To finish the proof of Theorem 1.10, we prove the following crucial result.

Lemma 5.14. Let $r \geqslant-1$. For every $\mathcal{T} \in \hat{\mathbb{G}}_{N}^{r+1}$,

$$
\text { Cone }\left[L \pi_{i}^{*} R \pi_{i *} L f_{i}{ }^{*} \mathcal{T} \rightarrow L f_{i}^{*} \mathcal{T}\right]
$$

belongs to the subcategory generated by $\hat{\mathbb{G}}_{N}^{r}$.

We postpone the proof of Lemma 5.14 to the end of this section. We use Lemma 5.14 to prove the following result (that implies Theorem 1.10).

Proposition 5.15. If $N \neq \emptyset$, the subcategory $D_{\text {cusp }}^{b}\left(X_{N}^{r}\right)$ is generated by $\hat{\mathbb{G}}_{N}^{r}$.

This proves the following theorem.

TheOREM 5.16. For all $r \geqslant-1$, the set $\hat{\mathbb{G}}_{N}^{r}$ is a full exceptional collection in $D_{\text {cusp }}^{b}\left(X_{N}^{r}\right)$.

In particular, when $r=-1$, this gives that the set $\hat{\mathbb{G}}_{N}$ is a full exceptional collection in $D_{\text {cusp }}^{b}\left(\overline{L M}_{N}\right)$ (Theorem 1.10).

Proof of Proposition 5.15. We argue by induction on the dimension $n+r$ and for a fixed $n+r$, by induction on $n$. The base of induction is $X_{1}^{r-1}$. Note that we have a $\mathbb{P}^{1}$-bundle $\pi_{1}: X_{1}^{r-1} \rightarrow \mathbb{P}^{r-1}$. By Orlov's Theorem 3.3, the category $D_{\text {cusp }}^{b}\left(X_{1}^{r-1}\right)$ is generated by

$$
\pi_{1}^{*} D^{b}\left(\mathbb{P}^{r-1}\right) \otimes \mathcal{O}\left(-E_{1}\right)=\left\langle\mathcal{O}\left(-r H+(r-1) E_{1}\right), \ldots, \mathcal{O}\left(-2 H+E_{1}\right), \mathcal{O}(-H)\right\rangle,
$$

which is precisely our claim in this case.

Assume $n \geqslant 2$. Choose an object $E \in D_{\text {cusp }}^{b}\left(X_{N}^{r}\right)$ such that $R \operatorname{Hom}(\mathcal{T}, E)=0$ for every $\mathcal{T} \in \hat{\mathbb{G}}_{N}^{r}$. We need to show that $E=0$. We first show that $R f_{i_{*}} E=0$ for all $i \in N$. Let $i \in N$. By Lemma 5.7, we have $R f_{i_{*}} E \in D_{\text {cusp }}^{b}\left(X_{N \backslash\{i\}}^{r+1}\right)$. By the inductive assumption, to prove that $R f_{i_{*}} E=0$, it is sufficient to prove that $R \operatorname{Hom}\left(\mathcal{T}, R f_{i_{*}} E\right)=0$ for every $\mathcal{T} \in \hat{\mathbb{G}}_{N \backslash\{i\}}^{r+1}$. Note that

$$
R \operatorname{Hom}\left(\mathcal{T}, R f_{i_{*}} E\right)=R \operatorname{Hom}\left(L f_{i}^{*} \mathcal{T}, E\right) .
$$

If we let $C=$ Cone $\left[L \pi_{i}{ }^{*} R \pi_{i *} L f_{i}{ }^{*} \mathcal{T} \rightarrow L f_{i}{ }^{*} \mathcal{T}\right]$, it follows by Lemma 5.14 that $R \operatorname{Hom}(C, E)=0$. Using the distinguished triangle

$$
L \pi_{i}{ }^{*} R \pi_{i *} L f_{i}{ }^{*} \mathcal{T} \rightarrow L f_{i}^{*} \mathcal{T} \rightarrow C \rightarrow L \pi_{i}{ }^{*} R \pi_{i *} L f_{i}{ }^{*} \mathcal{T}[1]
$$




\section{DERIVED CATEGORY OF MODUli OF POINTED CURVES. I}

and the fact that for all $F \in D^{b}\left(X_{N \backslash\{i\}}^{r}\right)$, we have (since $E \in D_{\text {cusp }}^{b}\left(X_{N}^{r}\right)$ )

$$
R \operatorname{Hom}\left(L \pi_{i}^{*} F, E\right)=R \operatorname{Hom}\left(F, R \pi_{i *} E\right)=0,
$$

it follows that $R \operatorname{Hom}\left(L f_{i}^{*} \mathcal{T}, E\right)=0$. This proves that $R f_{i *} E=0$ for all $i \in N$. In particular, by Lemma 3.1, the support Supp $E$ of $E$ is contracted by all birational maps $f_{i}$, for $i \in N$ :

$$
\operatorname{Supp} E \subseteq \operatorname{Exc}\left(f_{1}\right) \cap \cdots \cap \operatorname{Exc}\left(f_{n}\right) .
$$

Since $\operatorname{Exc}\left(f_{i}\right)=\bigcup_{i \in S} E_{S}$ and $E_{S} \cap E_{T} \neq \emptyset$ if and only if $S \subseteq T$ or $T \subseteq S$, this implies that $\operatorname{Exc}\left(f_{1}\right) \cap \cdots \cap \operatorname{Exc}\left(f_{n}\right)$ can be non-empty only if $r \geqslant 1$, in which case this intersection is contained in $E_{N}$, the exceptional divisor corresponding to blowing up the proper transform $\Delta_{N} \cong \overline{L M}_{N}$ of the subspace spanned by the points in $N$ (the last blow-up). It follows that

$$
\operatorname{Supp} E \subseteq E_{N} \cong \Delta_{N} \times \mathbb{P}^{r} .
$$

For every $i \in N$, we can decompose $f_{i}=f_{i}^{\prime} \circ p$, where $p: X_{N}^{r} \rightarrow Y$ blows down $E_{N}$ (with image $\Delta_{N}$ ) and $f_{i}^{\prime}: Y \rightarrow X_{N \backslash\{i\}}^{r+1}$ is the composition of blow-downs of $E_{S}$ for $S \subsetneq N$ containing $i$. If we write $E_{S}^{\prime}=p\left(E_{S}\right)$, it is still the case that $E_{S}^{\prime} \cap E_{T}^{\prime} \neq \emptyset$ if and only if $S \subseteq T$ or $T \subseteq S$. It follows that

$$
\operatorname{Exc}\left(f_{1}^{\prime}\right) \cap \cdots \cap \operatorname{Exc}\left(f_{n}^{\prime}\right)=\emptyset .
$$

Since $R f_{i *}^{\prime} R p_{*} E=R f_{i_{*}} E=0$ for all $i$, equation(5.3) and Lemma 3.1 imply that $R p_{*} E=0$. Let $\alpha: E_{N} \hookrightarrow X_{N}^{r}$ be the inclusion map. By Orlov's Theorem 3.4, the object $E$ belongs to the subcategory in $D^{b}\left(X_{N}^{r}\right)$ with semi-orthogonal decomposition

$$
\left\langle R \alpha_{*}\left[D^{b}\left(\overline{L M}_{N}\right) \otimes \mathcal{O}_{\mathbb{P}^{r}}(-r)\right], \ldots, R \alpha_{*}\left[D^{b}\left(\overline{L M}_{N}\right) \otimes \mathcal{O}_{\mathbb{P}^{r}}(-1)\right]\right\rangle .
$$

In particular, there exist morphisms

$$
0=E_{0} \rightarrow E_{1} \rightarrow \cdots \rightarrow E_{r}=E
$$

that fit into exact triangles

$$
E_{i-1} \rightarrow E_{i} \rightarrow F_{i} \rightarrow E_{i-1}[1] \quad \text { with } \quad F_{i} \in R \alpha_{*}\left[D^{b}\left(\overline{L M}_{N}\right) \otimes \mathcal{O}_{\mathbb{P}^{r}}(-i)\right] .
$$

Claim 5.17. We have $F_{i} \in R \alpha_{*}\left[D_{\text {cusp }}^{b}\left(\overline{L M}_{N}\right) \otimes \mathcal{O}_{\mathbb{P}^{r}}(-i)\right]$ for all $1 \leqslant i \leqslant r$.

The proposition now follows immediately from Claim 5.17: by the inductive hypothesis, the subcategory $R \alpha_{*}\left[D_{\text {cusp }}^{b}\left(\overline{L M}_{N}\right) \otimes \mathcal{O}_{\mathbb{P}^{r}}(-i)\right]$ is generated by sheaves that belong to $\hat{\mathbb{G}}_{N}^{r}$, but the latter have no non-zero morphisms into $E$. Thus $E=0$.

Proof of Claim 5.17. Let $F_{i}=R \alpha_{*}\left(H_{i} \otimes \mathcal{O}_{\mathbb{P}^{r}}(-i)\right)$ for some $H_{i} \in D^{b}\left(\overline{L M}_{N}\right)$. We have to show that $H_{i} \in D_{\text {cusp }}^{b}\left(\overline{L M}_{N}\right)$ for all $i$.

Let $j \in N$, and let $\alpha_{j}: \overline{L M}_{N \backslash\{j\}} \times \mathbb{P}^{r} \hookrightarrow X_{N \backslash\{j\}}^{r}$ be the inclusion. Then

$$
R \pi_{j_{*}} F_{i}=R \pi_{j_{*}} R \alpha_{*}\left(H_{i} \otimes \mathcal{O}_{\mathbb{P}^{r}}(-i)\right)=R \alpha_{j_{*}}\left(R \pi_{j_{*}} H_{i} \otimes \mathcal{O}_{\mathbb{P}^{r}}(-i)\right),
$$

where $R \pi_{j_{*}} H_{i} \in D^{b}\left(\overline{L M}_{N \backslash\{j\}}\right)$. In particular, $R \pi_{j_{*}} H_{i}=0$ if and only if $R \pi_{j_{*}} F_{i}=0$. Note that $R \pi_{j_{*}} F_{i}$ belongs to the subcategory

$$
R \alpha_{j_{*}}\left[D^{b}\left(\overline{L M}_{N \backslash\{j\}}\right) \otimes \mathcal{O}_{\mathbb{P}^{r}}(-i)\right] .
$$

Suppose $R \pi_{j_{*}} F_{p} \neq 0$ for some $j \in N$, and choose the maximal $p$ with this property. Applying $R \pi_{j_{*}}$ to the filtration gives morphisms

$$
0=R \pi_{j_{*}} E_{0} \rightarrow R \pi_{j_{*}} E_{1} \rightarrow \cdots \rightarrow R \pi_{j_{*}} E_{r}=R \pi_{j_{*}} E=0
$$




\section{A.-M. Castravet and J. Tevelev}

that fit into exact triangles

$$
R \pi_{j_{*}} E_{i-1} \rightarrow R \pi_{j_{*}} E_{i} \rightarrow R \pi_{j_{*}} F_{i} \rightarrow R \pi_{j_{*}} E_{i-1}[1] .
$$

In particular, $R \pi_{j_{*}} E_{p}=R \pi_{j_{*}} E_{p+1}=\cdots=R \pi_{j_{*}} E_{r}=R \pi_{j_{*}} E=0$ and $R \pi_{j_{*}} F_{p} \simeq R \pi_{j_{*}} E_{p-1}[1]$. However, $R \pi_{j_{*}} E_{p-1}[1]$ belongs to the subcategory generated by

$$
R \alpha_{j} *\left[D^{b}\left(\overline{L M}_{N^{\prime}}\right) \otimes \mathcal{O}_{\mathbb{P}^{r}}(-i)\right]
$$

for $i<p$ and thus cannot have a non-zero morphism to $R \pi_{j_{*}} F_{p}$.

We now prove Lemma 5.14. The proof occupies the rest of this section. We first prove the case when $\mathcal{T}^{\prime}=G_{a}^{r+1 \vee}$ on $X_{N \backslash\{i\}}^{r+1}$ in Lemma 5.18.

Lemma 5.18. Let $r \geqslant-1$. For all $1 \leqslant a \leqslant n+r$ and $i \in N$, we have

$$
\begin{gathered}
\pi_{i *}\left(f_{i}^{*} G_{a}^{r+1 \vee}\right)=0, \\
R^{1} \pi_{i *}\left(f_{i}^{*} G_{a}^{r+1 \vee}\right)= \begin{cases}G_{a-1}^{r} \vee \oplus G_{a-2}^{r} \vee \oplus \cdots \oplus G_{1}^{r \vee} & \text { if } a \geqslant 2, \\
0 & \text { if } a=1,\end{cases} \\
\text { Cone }\left[L \pi_{i}^{*} R \pi_{i *} L f_{i}^{*} G_{a}^{r+1 \vee} \rightarrow L f_{i}^{*} G_{a}^{r+1}\right]=G_{a}^{r \vee} \oplus G_{a-1}^{r} \vee \oplus \cdots \oplus G_{1}^{r \vee} .
\end{gathered}
$$

Proof. If $a=1$, then $f_{i}^{*} G_{1}^{r+1 \vee}=-H=G_{1}^{r \vee}$ and the statements follow at once as $G_{1}^{r \vee}$ is a cuspidal object. Now, assume $a \geqslant 2$. For clarity, first consider the situation when $a \leqslant n$. In this case, we have

$$
\left(G_{a}^{r}\right)^{\vee}=-a H+(a-1) \sum_{j \in N \backslash i} E_{j}+\cdots+1 \cdot \sum_{J \subseteq N \backslash i,|J|=a-1} E_{J} .
$$

Define divisors on $X_{N}^{r}$ as follows:

$$
\begin{gathered}
E^{s}=\sum_{i \in I \subseteq N,|I|=s} E_{I} \quad(1 \leqslant s \leqslant a-1), \\
F_{s}=E^{1}+E^{2}+\cdots+E^{s}=E_{i}+\sum_{j} E_{i j}+\sum_{j, k} E_{i j k}+\cdots+\sum_{i \in I \subseteq N,|I|=s} E_{I}, \\
H_{1}=f_{i}^{*} G_{a}^{r+1}, \quad H_{2}=H_{1}+F_{1}, \quad H_{3}=H_{2}+F_{2}, \quad \cdots \quad, \quad H_{a}=H_{a-1}+F_{a-1}=G_{a}^{r \vee .} .
\end{gathered}
$$

There are two sets of exact sequences that we will use, identifying as usual divisors with the corresponding line bundles:

(A)

$$
\begin{gathered}
0 \rightarrow H_{1} \rightarrow H_{2} \rightarrow H_{2 \mid F_{1}} \rightarrow 0, \\
0 \rightarrow H_{2} \rightarrow H_{3} \rightarrow H_{3 \mid F_{2}} \rightarrow 0, \\
\vdots \\
0 \rightarrow H_{a-1} \rightarrow H_{a} \rightarrow H_{a \mid F_{a-1}} \rightarrow 0 .
\end{gathered}
$$

(B) For $2 \leqslant k \leqslant a-1$ and $1 \leqslant s \leqslant k-1$, letting

$$
Q_{s+1}^{k}=\left(H_{k+1}-F_{s}\right)_{\mid E^{s+1}}=\bigoplus_{i \in I \subseteq N,|I|=s+1}\left(H_{k+1}-F_{s}\right)_{\mid E_{I}},
$$




\section{DERIVED CATEGORY OF MODULI OF POINTED CURVES. I}

we have exact sequences

$$
\begin{gathered}
0 \rightarrow Q_{2}^{k} \rightarrow H_{k+1 \mid F_{2}} \rightarrow H_{k+1 \mid F_{1}} \rightarrow 0 \\
0 \rightarrow Q_{3}^{k} \rightarrow H_{k+1 \mid F_{3}} \rightarrow H_{k+1 \mid F_{2}} \rightarrow 0, \\
\vdots \\
0 \rightarrow Q_{k}^{k} \rightarrow H_{k+1 \mid F_{k}} \rightarrow H_{k+1 \mid F_{k-1}} \rightarrow 0 .
\end{gathered}
$$

Note that $F_{s+1}=F_{s}+E^{s+1}$ and that the sequences of type (B) are obtained by tensoring with $H_{k+1}$ the canonical sequence

$$
0 \rightarrow \mathcal{O}\left(-F_{s}\right)_{\mid E^{s+1}} \rightarrow \mathcal{O}_{F_{s+1}} \rightarrow \mathcal{O}_{F_{s}} \rightarrow 0 .
$$

Lemma 5.18 follows at once from taking $k=1$ in parts (A1) and (A2) in Claim 5.19. Parts (B1)-(B3) in the claim refer to the exact sequences of type (B), while parts (A1)-(A2) in the claim refer to the exact sequences of type (A). Parts (B1)-(B3) will be used to prove parts (A1)-(A2) (this is why they appear first).

We now discuss the case when $a>n$. In this case, we have

$$
\left(G_{a}^{r}\right)^{\vee}=-a H+(a-1) \sum_{j \in N \backslash i} E_{j}+\cdots+(a-(n-1)) E_{N \backslash i} .
$$

We define $F_{s}$ as above in the range $1 \leqslant s \leqslant n$ and let

$$
F_{a-1}=\cdots=F_{n+1}=F_{n}=E^{1}+E^{2}+\cdots+E^{n} .
$$

We define $H_{k}$ as above, for all $1 \leqslant k \leqslant a$. As before, $H_{a}=\left(G_{a}^{r}\right)^{\vee}$. We use the exact sequences of type $(\mathrm{A})$. In order to analyze the sheaves $H_{k+1 \mid F_{k}}$, there are two cases to consider: (1) $1 \leqslant k \leqslant n<a$ and (2) $n<k \leqslant a-1$. For a fixed $k$, we consider the sequences (5.4) of type (B), where for $1 \leqslant s \leqslant k-1$, the quotient $Q_{s+1}^{k}$ is defined as before if $s \leqslant n-1$, while if $k-1 \geqslant s \geqslant n$, we let

$$
Q_{n+1}^{k}=\cdots=Q_{k}^{k}=0 .
$$

Hence, the exact sequences of type (B) that we consider are

$$
\begin{gathered}
0 \rightarrow Q_{2}^{k} \rightarrow H_{k+1 \mid F_{2}} \rightarrow H_{k+1 \mid F_{1}} \rightarrow 0, \\
0 \rightarrow Q_{3}^{k} \rightarrow H_{k+1 \mid F_{3}} \rightarrow H_{k+1 \mid F_{2}} \rightarrow 0, \\
\vdots \\
0 \rightarrow Q_{n}^{k} \rightarrow H_{k+1 \mid F_{n}} \rightarrow H_{k+1 \mid F_{n-1}} \rightarrow 0 .
\end{gathered}
$$

The rest of the proof is identical, as Claim 5.19 still holds.

Claim 5.19. (B1) For $2 \leqslant k \leqslant a-1$ and $1 \leqslant s \leqslant \min \{k-1, n-1\}$, we have

$$
R \pi_{i *} Q_{s+1}^{k}=0 .
$$

(B2) For all $1 \leqslant s \leqslant k \leqslant a-1$, we have

$$
R^{l} \pi_{i *}\left(H_{k+1 \mid F_{s}}\right)=0 \text { for all } l>0 \text { and } \pi_{i *}\left(H_{k+1 \mid F_{s}}\right)=\left(G_{k}^{r}\right)^{\vee} \text {. }
$$

(B3) For all $1 \leqslant s \leqslant k \leqslant a-1$, the canonical map

$$
\pi_{i}^{*} \pi_{i *}\left(H_{k+1 \mid F_{s}}\right) \rightarrow\left(H_{k+1 \mid F_{s}}\right)
$$




\section{A.-M. Castravet and J. Tevelev}

is surjective with kernel $\pi_{i}^{*}\left(G_{k}^{r}\right)^{\vee} \otimes \mathcal{O}\left(-F_{s}\right)$. Moreover,

$$
\text { Cone }\left[L \pi_{i}^{*} R \pi_{i *}\left(H_{k+1 \mid F_{s}}\right) \rightarrow\left(H_{k+1 \mid F_{s}}\right)\right]=\left(\pi_{i}^{*}\left(G_{k}^{r}\right)^{\vee} \otimes \mathcal{O}\left(-F_{s}\right)\right)[1] .
$$

In particular,

$$
\text { Cone }\left[L \pi_{i}^{*} R \pi_{i *}\left(H_{k+1 \mid F_{k}}\right) \rightarrow\left(H_{k+1 \mid F_{k}}\right)\right]=\left(G_{k}^{r}\right)^{\vee}[1] .
$$

(A1) For all $1 \leqslant k \leqslant a-1$, we have

$$
\pi_{i *}\left(H_{k}\right)=0 \quad \text { and } \quad R^{1} \pi_{i *}\left(H_{k}\right)=\left(G_{a-1}^{r}\right)^{\vee} \oplus\left(G_{a-2}^{r}\right)^{\vee} \oplus \cdots \oplus\left(G_{k}^{r}\right)^{\vee} .
$$

(A2) For all $1 \leqslant k \leqslant a$, we have

$$
\text { Cone }\left[L \pi_{i}^{*} R \pi_{i *}\left(H_{k}\right) \rightarrow\left(H_{k}\right)\right]=\left(G_{a}^{r}\right)^{\vee} \oplus\left(G_{a-1}^{r}\right)^{\vee} \oplus \cdots \oplus\left(G_{k}^{r}\right)^{\vee} .
$$

Proof. We prove parts (B1)-(B3). From the commutative diagram

$$
\begin{gathered}
\overline{L M}_{I} \times X_{N \backslash I}^{r}=E_{I} \longrightarrow X_{N}^{r} \\
\left(\pi_{i}, \text { Id }\right) \downarrow \\
\overline{L M}_{J} \times X_{N \backslash I}^{r}=E_{J} \longrightarrow X_{N \backslash i}^{r},
\end{gathered}
$$

it follows that

$$
R \pi_{i *}\left(-\psi_{x} \otimes\left(G_{k-s}^{r}\right)^{\vee}\right)=R \pi_{i *}\left(-\psi_{x}\right) \otimes\left(G_{k-s}^{r}\right)^{\vee}=0
$$

as $R \pi_{i *}\left(-\psi_{x}\right)=0$. Hence, (5.20) implies that $R \pi_{i *} Q_{s+1}^{k}=0$, thus proving part (B1). Note that it suffices to prove parts (B2) and (B3) for $1 \leqslant s \leqslant \min \{k, n\}$, as $F_{n}=F_{n+1}=\cdots=F_{a-1}$. Clearly, part (B2) follows immediately from part (B1), the exact sequences of type (B) and the diagram (5.10). We now prove part (B3) by induction on $s$ (for a fixed $k$ ). Set

$$
h_{s}: \pi_{i}^{*} \pi_{i *}\left(H_{k+1 \mid F_{s}}\right) \rightarrow\left(H_{k+1 \mid F_{s}}\right), \quad \mathcal{K}_{s}=\operatorname{Ker}\left(h_{s}\right) .
$$

We use the following two observations: (1) for any sheaf $\mathcal{T}$, the canonical map $\pi_{i}{ }^{*} \pi_{i *}(\mathcal{T}) \rightarrow \mathcal{T}$ is non-zero whenever $\pi_{i *}(\mathcal{T})$ is non-zero, and (2) if $F \subset X$ is an effective divisor and $\mathcal{L}$ is a line bundle on $X$, the only non-zero morphism $\mathcal{L} \rightarrow \mathcal{L}_{\mid F}$ is the restriction map (with kernel $\mathcal{L}(-F)$ ).

When $s=1$, we have from part (B2) and (5.10) that

$$
\begin{aligned}
& \pi_{i *}\left(H_{k+1 \mid F_{1}}\right)=\left(G_{k}^{r}\right)^{\vee}, \quad \pi_{i}^{*} \pi_{i *}\left(H_{k+1 \mid F_{1}}\right)=\pi_{i}{ }^{*}\left(G_{k}^{r}\right)^{\vee}, \\
& H_{k+1 \mid F_{1}}=\left(G_{k}^{r}\right)^{\vee}=\left(\pi_{i}^{*}\left(G_{k}^{r}\right)^{\vee}\right)_{\mid F_{1}} \text { on } F_{1}=E_{i} .
\end{aligned}
$$

Hence, it follows from observations (1) and (2) that $h_{1}$ is surjective and $\mathcal{K}_{1}=\pi_{i}^{*}\left(G_{k}^{r}\right)^{\vee} \otimes \mathcal{O}\left(-F_{1}\right)$. Now assume that $h_{s}$ is surjective and $\mathcal{K}_{s}=\pi_{i}^{*}\left(G_{k}^{r}\right)^{\vee} \otimes \mathcal{O}\left(-F_{s}\right)$. By applying $\pi_{i}^{*} \pi_{i *}(-)$ to the exact sequence

$$
0 \rightarrow Q_{s+1}^{k} \rightarrow\left(H_{k+1}\right)_{\mid F_{s+1}} \rightarrow\left(H_{k+1}\right)_{\mid F_{s}} \rightarrow 0,
$$

it follows from part (B1) that there is a commutative diagram

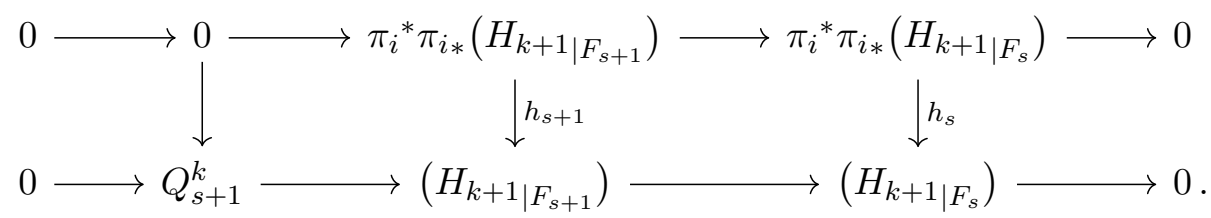

By our inductive assumption, $h_{s}$ is surjective. By the snake lemma, there is an exact sequence

$$
0 \rightarrow \mathcal{K}_{s+1} \rightarrow \mathcal{K}_{s} \rightarrow Q_{s+1}^{k} \rightarrow \operatorname{Coker}\left(h_{s+1}\right) \rightarrow 0 .
$$




\section{DERIVED CATEGORY OF MODUli OF POINTED CURVES. I}

The induced $\operatorname{map} \mathcal{K}_{s} \rightarrow Q_{s+1}^{k}$ is non-zero. Otherwise, $Q_{s+1}^{k} \cong \operatorname{Coker}\left(h_{s+1}\right)$, which implies that the exact sequence (5.6) is split since there is a retract $\left(H_{k+1 \mid F_{s+1}}\right) \rightarrow Q_{s+1}^{k}$. But the sequence (5.6) is obtained by tensoring the canonical sequence (5.4) with a line bundle, and (5.4) is not split, as there are no non-zero morphisms $\mathcal{O}_{F_{s+1}} \rightarrow \mathcal{O}_{E^{s+1}}\left(-F_{s}\right)$ :

$$
\operatorname{Hom}\left(\mathcal{O}_{F_{s+1}}, \mathcal{O}_{E^{s+1}}\left(-F_{s}\right)\right)=\mathrm{H}^{0}\left(\mathcal{O}_{E^{s+1}}\left(-F_{s}\right)\right)=0
$$

by (5.7), and we have a contradiction. We have $\mathcal{K}_{s}=\pi_{i}^{*}\left(G_{k}^{r}\right)^{\vee} \otimes \mathcal{O}\left(-F_{s}\right)$ by the induction assumption. By (5.20), we have $Q_{s+1}^{k}=\left(\pi_{i}^{*}\left(G_{k}^{r}\right)^{\vee} \otimes \mathcal{O}\left(-F_{s}\right)\right)_{\mid E^{s+1}}$. Hence, $Q_{s+1}^{k}=\left(\mathcal{K}_{s}\right)_{\mid E^{s+1}}$. By observation (2), the map $\mathcal{K}_{s} \rightarrow Q_{s+1}^{k}$ is surjective, that is, $\operatorname{Coker}\left(h_{s+1}\right)=0$, and furthermore

$$
\mathcal{K}_{s+1}=\mathcal{K}_{s}\left(-E^{s+1}\right)=\pi_{i}^{*}\left(G_{k}^{r}\right)^{\vee} \otimes \mathcal{O}\left(-F_{s}-E^{s+1}\right)=\pi_{i}^{*}\left(G_{k}^{r}\right)^{\vee} \otimes \mathcal{O}\left(-F_{s+1}\right) .
$$

This proves the first statement in part (B3). In particular, this gives

$$
\text { Cone }\left[L \pi_{i}^{*} R \pi_{i *}\left(H_{k+1 \mid F_{k}}\right) \rightarrow\left(H_{k+1 \mid F_{k}}\right)\right]=\left(\pi_{i}^{*}\left(G_{k}^{r}\right)^{\vee} \otimes \mathcal{O}\left(-F_{k}\right)\right)[1],
$$

and now the last statement in part (B3) follows from

$$
G_{k}^{r \vee}=\pi_{i}^{*}\left(G_{k}^{r}\right)^{\vee} \otimes \mathcal{O}\left(-F_{k}\right) .
$$

We now prove parts (A1) and (A2). Apply $\pi_{i *}(-)$ to the exact sequences of type (A). Then part (A1) follows from part (B2) and downward induction, using the fact that there are no nontrivial extensions between $\left(G_{k}^{r}\right)^{\vee}$ and $\left(G_{k^{\prime}}^{r}\right)^{\vee}$ for $k \neq k^{\prime}$. Similarly, to prove part (A2), we use downward induction on $1 \leqslant k \leqslant a$ and the exact sequences of type (A). As $H_{a}=\left(G_{a}^{r}\right)^{\vee}$, we have

$$
\text { Cone }\left[L \pi_{i}^{*} R \pi_{i *}\left(H_{a}\right) \rightarrow\left(H_{a}\right)\right]=\left(G_{a}^{r}\right)^{\vee} .
$$

Note that if $\pi: X \rightarrow Y$ is a morphism between smooth projective varieties and $0 \rightarrow A_{1} \rightarrow A_{2} \rightarrow$ $A_{3} \rightarrow 0$ is an exact sequence of sheaves on $X$, there is a distinguished triangle relating the cones $C_{i}=$ Cone $\left[L \pi^{*} R \pi_{*} A_{i} \rightarrow A_{i}\right]$ :

$$
C_{1} \rightarrow C_{2} \rightarrow C_{3} \rightarrow C_{1}[1]
$$

Then part (A2) follows from part (B3) by using the fact that there are no non-trivial extensions between $G_{k}^{r \vee}$ and $G_{k^{\prime}}^{r \vee}$ for $k \neq k^{\prime}$.

Claim 5.20. For all subsets $I \subseteq N$ with $i \in I$, where $|I|=s+1$ with $1 \leqslant s \leqslant n-1$, on $E_{I} \cong \overline{L M}_{I} \times X_{N \backslash I}^{r}$, we have

$$
F_{s \mid E_{I}}=\psi_{x} \otimes \mathcal{O}
$$

(here $x$ is the attaching point). Now, assume $1 \leqslant k \leqslant a-1$ and $1 \leqslant s \leqslant \min \{k-1, n-1\}$. Then

$$
H_{k+1 \mid E_{I}}=\mathcal{O} \otimes\left(G_{k-s}^{r}\right)^{\vee}=\left(\pi_{i}^{*}\left(G_{k}^{r}\right)^{\vee}\right)_{\mid E_{I}} .
$$

Hence, we have

$$
\begin{gathered}
\left(H_{k+1}-F_{s}\right)_{\mid E_{I}}=\left(-\psi_{x}\right) \otimes\left(G_{k-s}^{r}\right)^{\vee}=\left(\pi_{i}^{*}\left(G_{k}^{r}\right)^{\vee} \otimes \mathcal{O}\left(-F_{s}\right)\right)_{\mid E_{I}}, \\
Q_{s+1}^{k}=\bigoplus_{i \in I \subseteq N,|I|=s+1}\left(-\psi_{x}\right) \otimes\left(G_{k-s}^{r}\right)^{\vee}=\left(\pi_{i}^{*}\left(G_{k}^{r}\right)^{\vee} \otimes \mathcal{O}\left(-F_{s}\right)\right)_{\mid E^{s+1}} .
\end{gathered}
$$

Moreover, on $E_{i} \cong X_{N \backslash\{i\}}^{r}$, we have

$$
H_{k+1 \mid E_{i}}=\left(G_{k}^{r}\right)^{\vee} .
$$




\section{A.-M. Castravet and J. Tevelev}

Proof. To prove (5.7), we let $I=J \cup\{i\}$. Then $|J|=s$. We have

$$
\begin{aligned}
\left(F_{s}\right)_{\mid E_{I}} & =\left(E_{i}+\sum_{j \in N \backslash i} E_{i j}+\cdots+\sum_{K \subseteq N \backslash i,|K|=s-1} E_{K}\right)_{\mid E_{I}} \\
& =\delta_{J \cup\{x\}}+\sum_{j \in J} \delta_{(J \backslash\{j\}) \cup\{x\}}+\cdots+\sum_{j \in J} \delta_{j x}
\end{aligned}
$$

(as divisors on $\overline{L M}_{I}$ ). Using the $\psi_{x}$ Kapranov model of $\overline{L M}_{I}$, we have

$$
\left(F_{s}\right)_{\mid E_{I}}=\Lambda_{J}+\sum_{j \in J} E_{J \backslash\{j\}}+\cdots+\sum_{j \in J} E_{j}=H .
$$

Here $\Lambda_{J}$ denotes the class of the proper transform in $\overline{L M}_{I}$ of the hyperplane in $\mathbb{P}^{s}$ spanned by the points in $J$. This proves (5.7).

To see (5.8) and (5.10), recall that if $1 \leqslant k \leqslant a-1$, then

$$
\begin{gathered}
H_{k+1}=H_{1}+F_{1}+\cdots+F_{k}, \\
H_{1}=-a H+(a-1) \sum_{j \in N \backslash\{i\}} E_{j}+(a-2) \sum_{j, k \in N \backslash\{i\}} E_{j k}+\cdots+(a-t) \sum_{K \subseteq N \backslash\{i\},|K|=t} E_{K},
\end{gathered}
$$

where $t=\min \{a-1, n-1\}$. There are two cases to consider:

(1) $k \leqslant n$ (with either $n \leqslant a-1$ or $a-1 \leqslant n$ )

(2) $k>n$, in which case we must have $a-1>n$ and $t=n-1$. Note that we must have $r \geqslant 2$ as $n+r-1 \geqslant a-1>n$.

In case $(1)$, we have

$$
F_{1}+\cdots+F_{k}=k E_{i}+(k-1) \sum_{j \in N \backslash\{i\}} E_{i j}+\cdots+1 \cdot \sum_{i \in K \subseteq N,|K|=k} E_{K} .
$$

In case (2), we have

$$
\begin{aligned}
F_{1}+\cdots+F_{k}= & k E_{i}+(k-1) \sum_{j \in N \backslash\{i\}} E_{i j}+\cdots \\
& \cdots+(k-n+2) \sum_{i \in K \subseteq N,|K|=n-1} E_{K}+(k-n+1) E_{N} .
\end{aligned}
$$

Now let $1 \leqslant s \leqslant \min \{k-1, n-1\}$, and let $I \subseteq N$, with $i \in I$ and $|I|=s+1$ for some $0 \leqslant s \leqslant k-1$. Let

$$
\mathcal{O}\left(H_{k+1}\right)_{\mid E_{I}}=\mathcal{O}\left(H^{\prime}\right) \otimes \mathcal{O}\left(H^{\prime \prime}\right),
$$

where $H^{\prime}$ is the component on $\overline{L M}_{I}$ and $H^{\prime \prime}$ is the component on $X_{N \backslash I}^{r}$. We now compute $H^{\prime}$ and $H^{\prime \prime}$. Note that only the divisors $E_{K}$ with $I \subseteq K \subseteq N$ contribute to $H^{\prime \prime}$. For example, $H_{1}$ does not; that is, we have

$$
H^{\prime \prime}=\left(F_{1}+\cdots+F_{k}\right)_{\mid E_{I}}
$$




\section{DERIVED CATEGORY OF MODUli OF POINTED CURVES. I}

In case (1), we have

$$
\begin{aligned}
H^{\prime \prime}= & (k-s)\left(-\psi_{x}\right)+(k-s-1) \sum_{k \in N \backslash I} \delta_{k, x} \\
& +(k-s-2) \sum_{k, l \in N \backslash I} \delta_{k, l, x}+\cdots+1 \cdot \sum_{K \subseteq N \backslash I,|K|=k-s-1} \delta_{K \cup\{x\}} .
\end{aligned}
$$

(as a divisor on $\bar{M}_{0, N \backslash I}$ ), while in case (2), we have

$$
\begin{aligned}
H^{\prime \prime}= & (k-s)\left(-\psi_{x}\right)+(k-s-1) \sum_{k \in N \backslash I} \delta_{k, x} \\
& +(k-s-2) \sum_{k, l \in N \backslash I} \delta_{k, l, x}+\cdots+(k+1-n) \delta_{(N \backslash I) \cup\{x\}} .
\end{aligned}
$$

In both cases, $H^{\prime \prime}=\left(G_{k-s}^{r}\right)^{\vee}$ as by the definition of $G_{k-s}^{r}$ on $X_{N \backslash I}^{r}$,

$$
\left(G_{k-s}^{r}\right)^{\vee}=(k-s) H-(k-s-1) \sum_{j \in N \backslash I} E_{j}-\cdots-\left(k-s-t^{\prime}\right) \sum_{K \subseteq N \backslash I,|K|=t^{\prime}} E_{K}
$$

(in the Kapranov model given by $\psi_{x}$ ), where $t^{\prime}=\min \{k-s-1, n-s-1\}$; that is, $t^{\prime}=k-s-1$ in case (1), and $t^{\prime}=n-s-1$ in case (2).

We now calculate $H^{\prime}$. Let $I=J \cup\{i\}$. Since $|J|=s \leqslant \min \{k-1, n-1\}$, we have $s \leqslant t=$ $\min \{a-1, n-1\}$. Using the $\psi_{0}$ Kapranov model of $\overline{L M}_{I}$, we obtain that the contribution from $H_{1}$ to $H^{\prime}$ comes from

$$
-a H+(a-1) \sum_{j \in J} E_{j}+\cdots+(a-(s-1)) \sum_{K \subseteq J,|K|=s-1} E_{K}+(a-s) E_{J}
$$

and equals

$$
-a H+(a-1) \sum_{j \in J} E_{j}+\cdots+(a-(s-1)) \sum_{K \subseteq J,|K|=s-1} E_{K}+(a-s) \Lambda_{J},
$$

while the contribution from $F_{1}+\cdots+F_{k}$ to $H^{\prime}$ comes from

$$
\begin{aligned}
k E_{i} & +(k-1) \sum_{j \in J} E_{i j}+\cdots+(k-(s-2)) \sum_{K \subseteq J,|K|=s-2} E_{K \cup\{i\}} \\
& +(k-(s-1)) \sum_{K \subseteq J,|K|=s-1} E_{K \cup\{i\}}+(k-s) E_{J},
\end{aligned}
$$

and equals

$$
\begin{gathered}
k E_{i}+(k-1) \sum_{j \in J} E_{i j}+\cdots+(k-(s-2)) \sum_{K \subseteq J,|K|=s-2} E_{K \cup\{i\}} \\
+(k-(s-1)) \sum_{K \subseteq J,|K|=s-1} \Lambda_{K \cup\{i\}}+(k-s)\left(-\psi_{x}\right) .
\end{gathered}
$$

Here $\Lambda_{S}$ (for $S \subseteq I$ with $|S|=s$ ) denotes the class of the proper transform in $\overline{L M}_{I}$ of the hyperplane in $\mathbb{P}^{s}$ spanned by the points in $S$, that is,

$$
\Lambda_{S}=H-\sum_{K \subseteq S, 1 \leqslant|K| \leqslant s-1} E_{K} .
$$




\section{A.-M. Castravet and J. Tevelev}

We now sum up these two terms and compute the coefficient of $H$ to be

$$
-a+(a-s)+(k-s+1) s-(k-s) s=0 .
$$

Here we use that on $\overline{L M}_{I}$, the class of $\psi_{x}$ in the $\psi_{0}$ Kapranov model is

$$
\psi_{x}=s H-(s-1) \sum_{j \in I}-(s-2) \sum_{j, k \in I}-\cdots .
$$

Similarly, the coefficient of $E_{K}$ for $K \subseteq J$ with $|K|=l$ is

$$
(a-l)-(a-s)-(k-s+1)(s-l)+(k-s)(s-l)=0,
$$

while the coefficient of $E_{K \cup\{i\}}$ for $K \subseteq J$ with $|K|=l$ is

$$
(k-l)-(k-s+1)(s-l)+(k-s)(s-l-1)=0 .
$$

Hence, $H^{\prime}=0$ and $\mathcal{O}\left(H_{k+1}\right)_{\mid E_{I}}=\mathcal{O} \otimes\left(G_{k-s}^{r}\right)^{\vee}$.

To see that $\left(\pi_{i}^{*}\left(G_{k}^{r}\right)^{\vee}\right)_{\mid E_{I}}=\mathcal{O} \otimes\left(G_{k-s}^{r}\right)^{\vee}$, we use the commutative diagram (5.5). Note the equality of line bundles $\left(G_{k}^{r}\right)^{\vee}{ }_{\mid E_{J}}=\mathcal{O} \otimes\left(G_{k-s}^{r}\right)^{\vee}$ (Remark 5.10). This finishes the proof of (5.8). The case when $E_{I}=E_{i}$ corresponds to the case $s=0$, and the above computation shows (5.10). Clearly, (5.20) follows from (5.8) and (5.7).

To prove the general case of Lemma 5.14, we need the following.

Lemma 5.21. If $\pi_{i}: X_{N}^{r} \rightarrow X_{N \backslash i}^{r}$ is the forgetful map, then for all $1 \leqslant a \leqslant n+r-1$, the line bundle $\pi_{i}^{*}\left(G_{a}^{r}\right)^{\vee} \otimes \mathcal{O}\left(-E_{i}\right)$ belongs to the subcategory generated by $\hat{\mathbb{G}}_{N}^{r}$.

Proof. Let $t=\min \{a-1, n-1\}$. Keeping the notation of the proof of Lemma 5.18, consider the divisors $F_{s}$ on $X_{N}^{r}$, for $1 \leqslant s \leqslant t+1$, defined by

$$
F_{s}=E^{1}+E^{2}+\cdots+E^{s}=E_{i}+\sum_{j} E_{i j}+\sum_{j, k} E_{i j k}+\cdots+\sum_{i \in I \subseteq N,|I|=s} E_{I},
$$

and let

$$
L^{1}=\pi_{i}^{*}\left(G_{a}^{r}\right)^{\vee}-F_{1}, \quad L^{2}=\pi_{i}^{*}\left(G_{a}^{r}\right)^{\vee}-F_{2}, \quad \cdots \quad, \quad L^{t+1}=\pi_{i}^{*}\left(G_{a}^{r}\right)^{\vee}-F_{t+1} .
$$

We claim that $\pi_{i}^{*}\left(G_{a}^{r}\right)^{\vee}-F_{t+1}=\left(G_{a}^{r}\right)^{\vee}$. This is clear if one considers separately the two cases $a \leqslant n$ and $a>n$. For example, if $a>n$, then

$$
\left(G_{a}^{r}\right)^{\vee}=-a H+(a-1) \sum_{j \in N}+\cdots+(a-n) E_{N}=\pi_{i}^{*}\left(G_{a}^{r}\right)^{\vee}-F_{n} .
$$

We have to prove that $L^{1}$ belongs to the subcategory generated by $\hat{\mathbb{G}}_{N}^{r}$. We use the exact sequences

$$
\begin{gathered}
0 \rightarrow L^{2} \rightarrow L^{1} \rightarrow \bigoplus_{j \in N \backslash i}\left(L^{1}\right)_{\mid E_{i j}} \rightarrow 0, \\
0 \rightarrow L^{3} \rightarrow L^{2} \rightarrow \bigoplus_{j, k \in N \backslash i}\left(L^{2}\right)_{\mid E_{i j k}} \rightarrow 0, \\
\vdots \\
0 \rightarrow L^{t+1} \rightarrow L^{t} \rightarrow \bigoplus_{\substack{J \subseteq N \backslash\{i\},|J|=t}}\left(L^{t}\right)_{\mid E_{J \cup\{i\}}} \rightarrow 0 .
\end{gathered}
$$




\section{DERIVED CATEGORY OF MODULI OF POINTED CURVES. I}

Clearly, it is enough to prove that for all $1 \leqslant s \leqslant t$ and $J \subseteq N \backslash\{i\}$ with $|J|=s$, the sheaves $\left(L^{s}\right)_{\mid E_{J \cup\{i\}}}$ are in the subcategory generated by $\hat{\mathbb{G}}_{N}^{r}$. Note that $E_{J \cup\{i\}}$ is a massive stratum in $X_{N}^{r}$ as

$$
|J \cup\{i\}|=s+1 \geqslant 2, \quad|N \backslash J|+r=n-s+r>0
$$

since $s \leqslant t \leqslant n-1$ and when $r=-1$, we have $t=a-1$ and $a \leqslant n-1$.

As in (5.8), we have

while by (5.7), we have

$$
\left(\pi_{i}^{*} G_{a}^{r \vee}\right)_{\mid E_{J \cup\{i\}}}=\mathcal{O} \otimes\left(G_{a-s}^{r}\right)^{\vee}
$$

$$
\mathcal{O}\left(-F_{s}\right)_{\mid E_{J \cup\{i\}}}=\left(-\psi_{x}\right) \otimes \mathcal{O} .
$$

It follows that $\left(L^{s}\right)_{\mid E_{J \cup\{i\}}}$ is one of the objects in $\hat{\mathbb{G}}_{N}^{r}$, as it equals $\left(-\psi_{x}\right) \otimes\left(G_{a-s}^{r}\right)^{\vee}$.

Proof of Lemma 5.14. Consider the case when $\mathcal{T}$ is a torsion sheaf. Let

$$
\mathcal{T}=\left(i_{Z}\right)_{*} \mathcal{L}, \quad \mathcal{L}=G_{a_{1}}^{\vee} \otimes \cdots \otimes G_{a_{l-1}}^{\vee} \otimes G_{a_{l}}^{r+1} \vee
$$

where $Z=Z_{N_{1}, N_{2}, \ldots, N_{l}}$ is the massive stratum in $X_{N \backslash\{i\}}^{r+1}$ corresponding to a partition $N_{1} \sqcup \cdots \sqcup N_{l}$ of $N \backslash\{i\}$. Since $Z$ is massive, we have $\left|N_{t}\right| \geqslant 2$ for every $1 \leqslant t \leqslant l-1$ and $\left|N_{l}\right|+r+1>0$. The preimage $Z^{\prime}=f_{i}^{-1}(Z)$ is a massive stratum in $X_{N}^{r}$, and there is a commutative diagram

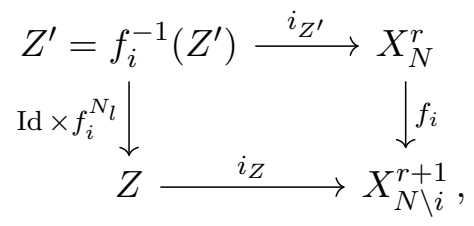

where $i_{Z^{\prime}}$ and $i_{Z}$ are the canonical inclusions, we identify

$$
Z=\overline{L M}_{N_{1}} \times \cdots \times \overline{L M}_{N_{l-1}} \times X_{N_{l}}^{r+1}, \quad Z^{\prime}=\overline{L M}_{N_{1}} \times \cdots \times \overline{L M}_{N_{l-1}} \times X_{N_{l} \cup\{i\}}^{r}
$$

and $f_{i}^{N_{l}}$ denotes the blow-up map $X_{N_{l} \cup\{i\}}^{r} \rightarrow X_{N_{l}}^{r+1}$ (we write $f_{i}$ whenever there is no risk of confusion). Let $\mathcal{T}^{\prime}=L f_{i}^{*} \mathcal{T}^{\prime}$. Then

$$
\mathcal{T}^{\prime}=\left(i_{Z^{\prime}}\right)_{*} \mathcal{L}^{\prime}, \quad \mathcal{L}^{\prime}=\left(\operatorname{Id} \times f_{i}\right)^{*} \mathcal{L}^{\prime}=G_{a_{1}}^{\vee} \otimes \cdots \otimes G_{a_{l-1}}^{\vee} \otimes f_{i}^{*} G_{a_{l}}^{r+1}{ }^{\vee} .
$$

We compute Cone $\left[L \pi_{i}{ }^{*} R \pi_{i *} \mathcal{T}^{\prime} \rightarrow \mathcal{T}^{\prime}\right]$ by the exact same argument as in the proof of Lemma 5.18. We define divisors $H_{1}, H_{2}, \ldots, H_{a_{l}}$ on $X_{N_{l} \cup\{i\}}^{r}$ exactly as before, so that we have

$$
H_{1}=f_{i}^{*}\left(G_{a_{l}}^{r+1}\right)^{\vee}, \quad H_{a_{l}}=\left(G_{a_{l}}^{r}\right)^{\vee} .
$$

On $X_{N_{l} \cup\{i\}}^{r}$, consider the exact sequences of type (A) in the proof of Lemma 5.18. After taking the box product with $G_{a_{1}}^{\vee} \otimes \cdots \otimes G_{a_{l-1}}^{\vee}$, one obtains exact sequences on $Z^{\prime}$. It is enough to prove that for all $1 \leqslant k \leqslant a_{l}-1$, the object Cone $\left[L \pi_{i}{ }^{*} R \pi_{i *} \mathcal{T}_{k} \rightarrow \mathcal{T}_{k}\right]$ is in the subcategory generated by $\hat{\mathbb{G}}_{N}^{r}$, where

$$
\mathcal{T}_{k}=\left(i_{Z^{\prime}}\right)_{*}\left(G_{a_{1}}^{\vee} \otimes \cdots \otimes G_{a_{l-1}}^{\vee} \otimes\left(H_{k+1 \mid F_{k}}\right)\right) .
$$

On $X_{N_{l} \cup\{i\}}^{r}$, we consider the exact sequences of type (B) in the proof of Lemma 5.18 after taking the box product with $G_{a_{1}}^{\vee} \cdots \otimes G_{a_{l-1}}^{\vee}$. Let

$$
\begin{gathered}
\mathcal{T}_{k, s}=\left(i_{Z^{\prime}}\right)_{*}\left(G_{a_{1}}^{\vee} \otimes \cdots \otimes G_{a_{l-1}}^{\vee} \otimes Q_{s+1}^{k}\right), \\
\tilde{\mathcal{T}}_{k}=\left(i_{Z^{\prime}}\right)_{*}\left(G_{a_{1}}^{\vee} \cdots \otimes G_{a_{l-1}}^{\vee} \otimes\left(H_{k+1 \mid F_{1}}\right)\right) .
\end{gathered}
$$




\section{A.-M. Castravet and J. Tevelev}

Then Cone $\left[L \pi_{i}{ }^{*} R \pi_{i *} \mathcal{T}_{k} \rightarrow \mathcal{T}_{k}\right]$ is in the subcategory generated by $\hat{\mathbb{G}}_{N}^{r}$ if and only if

$$
\text { Cone }\left[L \pi_{i}^{*} R \pi_{i *} \mathcal{T}_{k, s} \rightarrow \mathcal{T}_{k, s}\right] \quad \text { and } \quad \text { Cone }\left[L \pi_{i}{ }^{*} R \pi_{i *} \tilde{\mathcal{T}}_{k} \rightarrow \tilde{\mathcal{T}}_{k}\right]
$$

are in the subcategory generated by $\hat{\mathbb{G}}_{N}^{r}$. By (5.20), the sheaf $Q_{s+1}^{k}$ is a direct sum of objects in $\hat{\mathbb{G}}_{N_{l} \cup\{i\}}^{r}$. Hence, $\mathcal{T}_{k, s}$ is a direct sum of objects in $\hat{\mathbb{G}}_{N}^{r}$. In particular, Cone $\left[L \pi_{i}{ }^{*} R \pi_{i *} \mathcal{T}_{k, s} \rightarrow\right.$ $\left.\mathcal{T}_{k, s}\right]=\mathcal{T}_{k, s}$. We are left to prove that Cone $\left[L \pi_{i}{ }^{*} R \pi_{i *} \tilde{\mathcal{T}}_{k} \rightarrow \tilde{\mathcal{T}}_{k}\right]$ is in the subcategory generated by $\hat{\mathbb{G}}_{N}^{r}$.

For simplicity, set $\tilde{\mathcal{T}}=\tilde{\mathcal{T}}_{k}$. Let $\bar{Z}:=\pi_{i}\left(Z^{\prime}\right)$. We make the identification

$$
\bar{Z}=\overline{L M}_{N_{1}} \times \cdots \times \overline{L M}_{N_{l-1}} \times X_{N_{l}}^{r} .
$$

Then $\pi_{i}^{-1}(\bar{Z})=Z^{1} \cup \cdots \cup Z^{l}$, where

$$
\begin{gathered}
Z^{l}=Z^{\prime}=\overline{L M}_{N_{1}} \times \cdots \times \overline{L M}_{N_{l-1}} \times X_{N_{l} \cup\{i\}}^{r}, \\
Z^{t}=\overline{L M}_{N_{1}} \times \cdots \times \overline{L M}_{N_{t} \cup\{i\}} \times \cdots \times \overline{L M}_{N_{l-1}} \times X_{N_{l}}^{r}, \quad 1 \leqslant t \leqslant l-1 .
\end{gathered}
$$

As the divisor $E_{i}$ in $X_{N_{l} \cup\{i\}}^{r}$ can be identified with $\overline{L M}_{\{i\}} \times X_{N_{l}}^{r}$, the sheaf $\tilde{\mathcal{T}}$ is supported on the non-massive stratum

$$
\begin{gathered}
Z^{l} \cap Z^{l-1}=\overline{L M}_{N_{1}} \times \cdots \times \overline{L M}_{N_{l-1}} \times \overline{L M}_{\{i\}} \times X_{N_{l}}^{r}, \\
\tilde{\mathcal{T}}=\left(i_{Z^{l} \cap Z^{l-1}}\right)_{*} \mathcal{M}, \quad \mathcal{M}=G_{a_{1}}^{\vee} \times \cdots \otimes G_{a_{l-1}}^{\vee} \otimes \mathcal{O} \otimes G_{k}^{r \vee},
\end{gathered}
$$

where $i_{Z^{l} \cap Z^{l-1}}: Z^{l} \cap Z^{l-1} \rightarrow X_{N}^{r}$ is the canonical inclusion. Denote by

$$
v: Z^{l} \cap Z^{l-1} \rightarrow \pi_{i}^{-1}(\bar{Z}), \quad u: \pi_{i}^{-1}(\bar{Z}) \rightarrow X_{N}^{r}
$$

the canonical inclusions. Then $i_{Z^{l} \cap Z^{l-1}}=u \circ v$. Let $\rho=\pi_{i \mid \pi_{i}-1}(\bar{Z})$. There is a commutative diagram

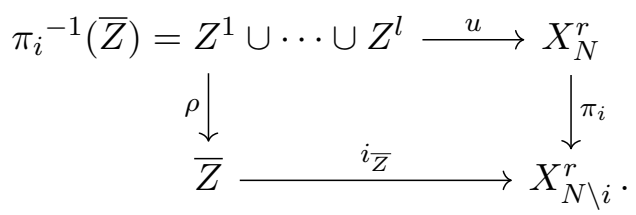

The restriction maps $\rho_{\mid Z^{t}}: Z^{t} \rightarrow \bar{Z}$ are induced by the forgetful maps $\overline{L M}_{N_{t} \cup\{i\}} \rightarrow \overline{L M}_{N_{t}}$ if $t<l$ and $X_{N_{l} \cup\{i\}}^{r} \rightarrow X_{N_{l}}^{r}$ for $t=l$. Note that the restriction map $\rho_{\mid Z^{l} \cap Z^{l-1}}: Z^{l} \cap Z^{l-1} \rightarrow \bar{Z}$ is an isomorphism. Let

$$
\overline{\mathcal{M}}=R \rho_{*}\left(R v_{*} \mathcal{M}\right)=G_{a_{1}}^{\vee} \otimes \cdots \otimes G_{a_{l-1}}^{\vee} \otimes G_{k}^{r \vee} .
$$

For all $1 \leqslant t \leqslant l-1$, we have

$$
\left(\rho^{*} \overline{\mathcal{M}}\right)_{\mid Z^{t}}=G_{a_{1}}^{\vee} \otimes \cdots \otimes \pi_{i}^{*}\left(G_{a_{t}}^{r \vee}\right) \cdots \otimes G_{a_{l-1}}^{\vee} \otimes G_{k}^{r \vee}
$$

while

$$
\left(\rho^{*} \overline{\mathcal{M}}\right)_{\mid Z^{l}}=G_{a_{1}}^{\vee} \otimes \cdots \otimes G_{a_{t}}^{r \vee \cdots \otimes} G_{a_{l-1}}^{\vee} \otimes \pi_{i}^{*}\left(G_{k}^{r \vee}\right)
$$

The strata $Z^{1}, \ldots, Z^{l}$ intersect: if $t<s$, then $Z_{t} \cap Z_{s} \neq \emptyset$ if and only if $s=t+1$. There are 


\section{DERIVED CATEGORY OF MODULI OF POINTED CURVES. I}

exact sequences

$$
\begin{aligned}
0 & \rightarrow \mathcal{O}_{Z^{1} \cup \cdots \cup Z^{l-1}}\left(-Z^{l}\right) \rightarrow \mathcal{O}_{Z^{1} \cup \cdots \cup Z^{l}} \rightarrow \mathcal{O}_{Z^{l}} \rightarrow 0 \\
0 & \rightarrow \mathcal{O}_{Z^{1} \cup \cdots \cup Z^{l-2}}\left(-Z^{l-1}\right) \rightarrow \mathcal{O}_{Z^{1} \cup \cdots \cup Z^{l-1}}\left(-Z^{l}\right) \rightarrow \mathcal{O}_{Z^{l-1}}\left(-Z^{l}\right) \rightarrow 0 \\
\vdots & \\
0 & \rightarrow \mathcal{O}_{Z^{1}}\left(-Z^{2}\right) \rightarrow \mathcal{O}_{Z^{1} \cup Z^{2}}\left(-Z^{3}\right) \rightarrow \mathcal{O}_{Z^{2}}\left(-Z^{3}\right) \rightarrow 0 .
\end{aligned}
$$

We also consider the exact sequence

$$
0 \rightarrow \mathcal{O}_{Z^{l}}\left(-Z^{l-1}\right) \rightarrow \mathcal{O}_{Z^{l}} \rightarrow \mathcal{O}_{Z^{l} \cap Z^{l-1}} \rightarrow 0 .
$$

We tensor all the above sequences with $\rho^{*} \overline{\mathcal{M}}$. If we write

$$
\begin{array}{cl}
\mathcal{N}^{t}=\rho^{*} \overline{\mathcal{M}} \otimes \mathcal{O}_{Z^{t}}\left(-Z^{t+1}\right) \quad(1 \leqslant t \leqslant l-1), & \mathcal{N}^{0}=\rho^{*} \overline{\mathcal{M}} \otimes \mathcal{O}_{Z^{l}}\left(-Z^{l-1}\right), \\
\mathcal{F}^{t}=\rho^{*} \overline{\mathcal{M}} \otimes \mathcal{O}_{Z^{1} \cup \ldots \cup Z^{t}}\left(-Z^{t+1}\right) & (1 \leqslant t \leqslant l-1),
\end{array}
$$

then we have exact sequences on $Z^{1} \cup \cdots \cup Z^{l}$

$$
\begin{gathered}
0 \rightarrow \mathcal{F}^{l-2} \rightarrow \rho^{*} \overline{\mathcal{M}} \rightarrow\left(\rho^{*} \overline{\mathcal{M}}\right)_{\mid Z^{l}} \rightarrow 0 \\
0 \rightarrow \mathcal{F}^{l-3} \rightarrow \mathcal{F}^{l-2} \rightarrow \mathcal{N}^{l-1} \rightarrow 0 \\
0 \rightarrow \mathcal{F}^{l-4} \rightarrow \mathcal{F}^{l-3} \rightarrow \mathcal{N}^{l-2} \rightarrow 0 \\
\vdots \\
0 \rightarrow \mathcal{F}^{1}=\mathcal{N}^{1} \rightarrow \mathcal{F}^{2} \rightarrow \mathcal{N}^{2} \rightarrow 0
\end{gathered}
$$

and, furthermore,

$$
0 \rightarrow \mathcal{N}^{0} \rightarrow\left(\rho^{*} \overline{\mathcal{M}}\right)_{\mid Z^{l}} \rightarrow v_{*} \mathcal{M} \rightarrow 0
$$

Consider the push-forwards via $u_{*}(-)$ to $X_{N}^{r}$ of all of the above exact sequences. Recall that $\tilde{\mathcal{T}}=u_{*}\left(v_{*} \mathcal{M}\right)$. To prove that Cone $\left[L \pi_{i}{ }^{*} R \pi_{i *} \tilde{\mathcal{T}} \rightarrow \tilde{\mathcal{T}}\right]$ is in the subcategory generated by $\hat{\mathbb{G}}_{N}^{r}$, it suffices to prove that for $\mathcal{N}$ among

$$
\rho^{*} \overline{\mathcal{M}}, \quad \mathcal{N}^{1}, \quad \ldots, \quad \mathcal{N}^{l-1}, \quad \mathcal{N}^{0},
$$

we have that Cone $\left[L \pi_{i}{ }^{*} R \pi_{i *}\left(u_{*} \mathcal{N}\right) \rightarrow\left(u_{*} \mathcal{N}\right)\right]$ is in the subcategory generated by $\hat{\mathbb{G}}_{N}^{r}$. This is clear for $\rho^{*} \overline{\mathcal{M}}$, as $u_{*} \rho^{*} \overline{\mathcal{M}}=\pi_{i}^{*} i \bar{Z}_{*} \overline{\mathcal{M}}$ (since $\pi_{i}$ is flat), and we have Cone $\left[L \pi_{i}^{*} R \pi_{i *} L \pi_{i}^{*} A \rightarrow\right.$ $\left.L \pi_{i}^{*} A\right]=0$ for any $A$. As

$$
\mathcal{O}_{Z^{t}}\left(-Z^{t+1}\right)=\mathcal{O}_{\overline{L M}_{N_{1}}} \otimes \cdots \otimes \mathcal{O}_{\overline{L M}_{N_{t}}}\left(-\delta_{i, y}\right) \otimes \cdots \otimes \mathcal{O}_{X_{N_{l}}^{r} \cup\{i\}},
$$

where $y$ is one of the attaching points of $\overline{L M}_{N_{t}}$, using (5.11) and Lemma 5.21, it follows that $u_{*} \mathcal{N}^{t}$ is in the subcategory generated by $\hat{\mathbb{G}}_{N}^{r}$. In particular,

$$
R \pi_{i *}\left(u_{*} \mathcal{N}^{t}\right)=0 \quad \text { and } \quad \text { Cone }\left[L \pi_{i}{ }^{*} R \pi_{i *}\left(u_{*} \mathcal{N}^{t}\right) \rightarrow\left(u_{*} \mathcal{N}^{t}\right)\right]=u_{*} \mathcal{N}^{t}
$$

Similarly, $u_{*} \mathcal{N}^{0}$ is in the subcategory generated by $\hat{\mathbb{G}}_{N}^{r}$ since

$$
\mathcal{O}_{Z^{l}}\left(-Z^{l-1}\right)=\mathcal{O}_{\overline{L M}_{N_{1}}} \otimes \cdots \otimes \mathcal{O}_{X_{N_{l} \cup\{i\}}^{r}}\left(-E_{i}\right)
$$

and we may use (5.12) and Lemma 5.21. 


\section{A.-M. Castravet and J. Tevelev}

\section{ACKNOWLEDGEMENTS}

We are grateful to Alexander Kuznetsov for a suggestion to think about the derived category of pointed curves in the equivariant setting and for several improvements of the exposition. We are grateful to Michel Brion for pointing out the connection to the wonderful compactification of $\mathrm{PGL}_{n}$. We thank Asher Auel, Chunyi Li, Daniel Halpern-Leistner, Emanuele Macrì and Dimitri Zvonkine for useful conversations. We are grateful to the referee for the careful reading and numerous very helpful comments.

Parts of this paper were written while the first author was visiting the Institut des Hautes Études Scientifiques in Bures-sur-Yvette, France and the second author was visiting the Fields Institute in Toronto, Canada.

\section{REFERENCES}

BFK19 M. Ballard, D. Favero and L. Katzarkov, Variation of geometric invariant theory quotients and derived categories, J. reine angew. Math. 746 (2019), 235-303; doi:10.1515/crelle-2015-0096.

BK05 M. Brion and S. Kumar, Frobenius splitting methods in geometry and representation theory, Progr. Math., vol. 231 (Birkhäuser Boston, Inc., Boston, MA, 2005); doi:10.1007/b137486.

BM13 J. Bergström and S. Minabe, On the cohomology of moduli spaces of (weighted) stable rational curves, Math. Z. 275 (2013), no. 3-4, 1095-1108; doi:10.1007/s00209-013-1171-8.

BM14 On the cohomology of the Losev-Manin moduli space, Manuscripta Math. 144 (2014), no. 1-2, 241-252; doi:10.1007/s00229-013-0647-5.

Bri07 M. Brion, The total coordinate ring of a wonderful variety, J. Algebra 313 (2007), no. 1, 61-99; doi:10.1016/j.jalgebra.2006.12.022.

CT12 A.-M. Castravet and J. Tevelev, Rigid curves on $\bar{M}_{0, n}$ and arithmetic breaks, in Compact Moduli Spaces and Vector Bundles, Contemp. Math., vol. 564 (Amer. Math. Soc., Providence, RI, 2012), 19-67; doi:10.1090/conm/564/11156.

CT13 , Hypertrees, projections, and moduli of stable rational curves, J. reine angew. Math. 675 (2013), 121-180; doi:10.1515/crelle.2011.189.

CT15 $-\bar{M}_{0, n}$ is not a Mori dream space, Duke Math. J. 164 (2015), no. 8, 1641-1667; doi: 10.1215/00127094-3119846.

Get95 E. Getzler, Operads and moduli spaces of genus 0 Riemann surfaces, The Moduli Space of Curves (Texel Island, 1994), Progr. Math., vol. 129 (Birkhäuser Boston, Boston, MA, 1995), 199-230; doi:10.1007/978-1-4612-4264-2_\{\}8.

Has03 B. Hassett, Moduli spaces of weighted pointed stable curves, Adv. Math. 173 (2003), no. 2, 316352; doi:10.1016/S0001-8708(02)00058-0.

Huy06 D. Huybrechts, Fourier-Mukai transforms in algebraic geometry, Oxford Math. Monogr. (The Clarendon Press, Oxford Univ. Press, Oxford, 2006); doi:10.1093/acprof:oso/ 9780199296866.001 .0001$.

Kee92 S. Keel, Intersection theory of moduli space of stable n-pointed curves of genus zero, Trans. Amer. Math. Soc. 330 (1992), no. 2, 545-574; doi:10.2307/2153922.

KT09 S. Keel and J. Tevelev, Equations for $\bar{M}_{0, n}$, Internat. J. Math. 20 (2009), no. 9, 1159-1184; doi:10.1142/S0129167X09005716.

Kuz08 A. Kuznetsov, Lefschetz decompositions and categorical resolutions of singularities, Selecta Math. (N.S.) 13 (2008), no. 4, 661-696; doi:10.1007/s00029-008-0052-1.

Kuz16 Derived categories view on rationality problems, in Rationality Problems in Algebraic Geometry, Lecture Notes in Math., vol. 2172 (Springer, Cham, 2016), 67-104; doi:10.1007/ 978-3-319-46209-7_3. 


\section{DERIVED CATEGORY OF MODULI OF POINTED CURVES. I}

LM00 A. Losev and Yu. Manin, New moduli spaces of pointed curves and pencils of flat connections, Michigan Math. J. 48 (2000), no. 1, 443-472; doi:10.1307/mmj/1030132728.

MP97 A.S. Merkurjev and I. A. Panin, $K$-theory of algebraic tori and toric varieties, $K$-Theory 12 (1997), no. 2, 101-143; doi:10.1023/A:1007770500046.

MS13 Yu. I. Manin and M. N. Smirnov, On the derived category of $\bar{M}_{0, n}$, Izv. Math. 77 (2013), no. 3, 525-540; doi:10.4213/im7944.

Orl93 D. Orlov, Projective bundles, monoidal transformations, and derived categories of coherent sheaves, Izv. Math. 41 (1993), no. 1, 133-141; doi:10.1070/IM1993v041n01ABEH002182.

Orl11_ Formal completions and idempotent completions of triangulated categories of singularities, Adv. Math. 226 (2011), no. 1, 206-217; doi:10.1016/j.aim.2010.06.016.

Sta20 The Stacks Project Authors, Stacks Project, version 2020, available at https://stacks.math. columbia.edu/.

Voi07 C. Voisin, Hodge theory and complex algebraic geometry. I, Cambridge Stud. Adv. Math., vol. 76 (Cambridge Univ. Press, Cambridge, 2007); doi:10.1017/CB09780511615344.

Ana-Maria Castravet ana-maria.castravet@uvsq.fr

Université Paris-Saclay, UVSQ, CNRS, Laboratoire de Mathématiques de Versailles, 78000, Versailles, France

Jenia Tevelev tevelev@math.umass.edu

Department of Mathematics and Statistics, University of Massachusetts Amherst, 710 North Pleasant Street, Amherst, MA 01003, USA

Laboratory of Algebraic Geometry and its Applications, HSE, Moscow, Russia 